\title{
Overhauser Dynamic Nuclear Polarization with Selectively Deuterated
}

\section{BDPA Radicals}

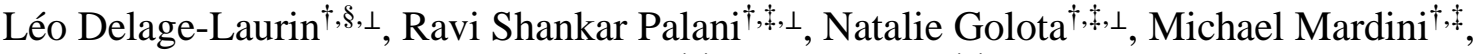 \\ Yifu Ouyang ${ }^{\dagger, \$}$, Kong O. Tan ${ }^{\dagger, *}$, \\ Timothy M. Swager ${ }^{\dagger, \&}$ and Robert G. Griffin ${ }^{\dagger, *}$ \\ †Department of Chemistry \\ and \\ FFrancis Bitter Magnet Laboratory \\ Massachusetts Institute of Technology \\ Cambridge, MA 02139, USA \\ ${ }^{\S}$ Institute for Soldier Nanotechnologies \\ Cambridge, MA 02139, USA \\ "Present address: Laboratoire des Biomolécules UMR 7203 \\ Département de Chimie \\ École Normale Supérieure \\ 75231 CEDEX 05, Paris, France \\ Supporting Information
}

\section{CONTENTS}

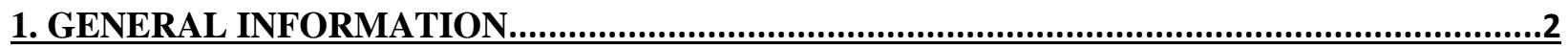

2. SYNTHESIS OF SELECTIVELY DEUTERATED FLUORENE MOIETIES …............................

3. SYNTHESIS OF SELECTIVELY DEUTERATED AND PERDEUTERATED BDPA

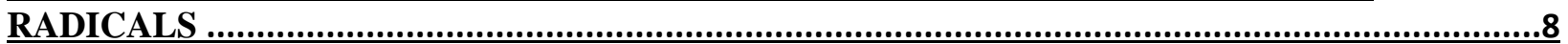

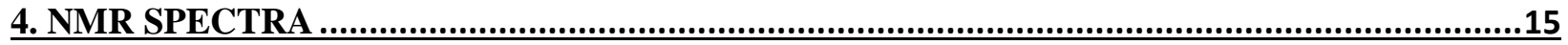

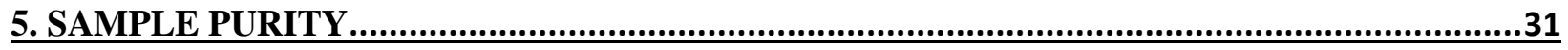

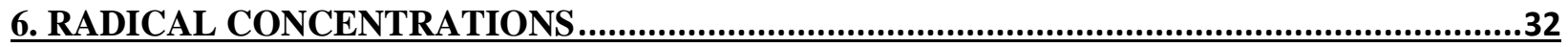




\section{General Information about Synthesis}

Unless otherwise stated, all reactions were carried out under an atmosphere of nitrogen using the standard Schlenk technique. All solvents and reagents were obtained from commercial sources and were purified following standard procedures before use if necessary.

NMR spectra were measured on Bruker Avance III HD $600 \mathrm{MHz}$ spectrometer. Proton chemical shifts are expressed in parts per million (ppm, $\delta$ scale) and are referenced to the residual proton in the NMR solvents $\mathrm{CDCl}_{3}$ at $\delta 7.26$ or DMSO- $d_{6}$ at $2.50 \mathrm{ppm} .{ }^{1} \mathrm{H}$ NMR spectroscopic data are reported as follows: Chemical shift in ppm (multiplicity, coupling constants $\mathrm{J}(\mathrm{Hz})$, integration intensity). The multiplicities are abbreviated with s (singlet), $d$ (doublet), $t$ (triplet), $m$ (multiplet). All ${ }^{13} \mathrm{C}$ spectra recorded are proton-decoupled. The carbon chemical shifts are expressed in parts per million (ppm, $\delta$ scale) and are referenced to the carbon resonance of the NMR solvents $\mathrm{CDCl}_{3}$ at $\delta 77.16$ or DMSO- $d_{6}$ at $39.52 \mathrm{ppm} .{ }^{13} \mathrm{C}$ NMR spectroscopic data are reported as follows: Chemical shift in ppm (multiplicity, coupling constants $\mathrm{J}(\mathrm{Hz})$ ). All raw fid files were processed and the spectra analyzed using the program MestReNOVA 14.2 from Mestrelab Supporting Information SI4 Research S. L.

Deuterium incorporation $<100 \%$ at specific positions will result in non-deuterated analogs of targeted compounds. These impurities will appear on NMR spectra and therefore need to be distinguished from the desired selectively deuterated compounds. Hence, for ${ }^{1} \mathrm{H}$ NMR, two set of signals are reported, one corresponding to the desired product, labelled Deuterated Product (DP), and the other corresponding to the non-deuterated impurity, labelled Non-Deuterated Impurity (NDI). Overlap between both products signals occurred, and the reported signals are from the peaks that could be resolved. Deuterium incorporation was calculated from the integrated ratio of well-defined ${ }^{1} \mathrm{H}$ NMR signals that are known to be associated with both compounds. For clarity purposes, ${ }^{13} \mathrm{C}$ NMR signals were only reported for the main product, but the signals from nondeuterated impurities can be observed in spectra with high signal-to-noise ratio (SNR). The chemical shifts of the deuterated products and non-deuterated impurities differ due to the ${ }^{1} \mathrm{H} /{ }^{2} \mathrm{H}$ isotope effect. Signals from carbon atoms directly attached to deuterium atoms are triplet or multiplet due to deuterium coupling. Due to low SNR or the complexity of the molecules further down the synthetic route, some signals could or should not be assigned as triplets, and were assigned as multiplets.

High-resolution mass spectra were recorded on a JEOL AccuTOF LC-Plus 46 with an ionSense DART system. 


\section{Synthesis of Selectively Deuterated Fluorene Moieties}<smiles>O=C1c2cc(O)ccc2-c2ccc(O)cc21</smiles>

1

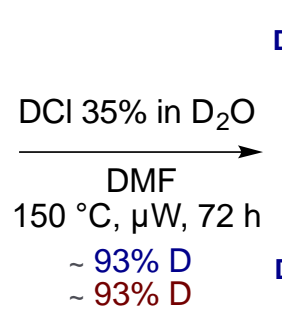

$95 \%$<smiles>[2H]c1cc2c(c([2H])c1O)C(=O)c1c([2H])c([2H])c(O)c([2H])c1-2</smiles>

2

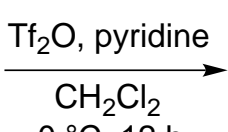

$0{ }^{\circ} \mathrm{C}, 12 \mathrm{~h}$ $92 \%$

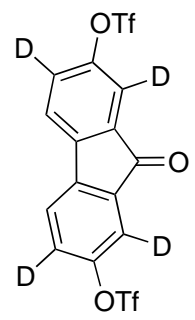

3
$9 H$-fluorene-1,3,6,8- $d_{4}$
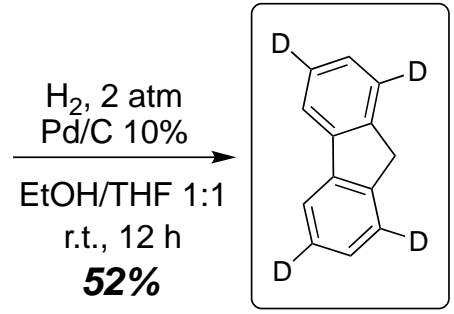

4

Figure S1. General synthetic route for $\mathbf{9 H}$-fluorene-1,3,6,8- $\boldsymbol{d}_{4}$
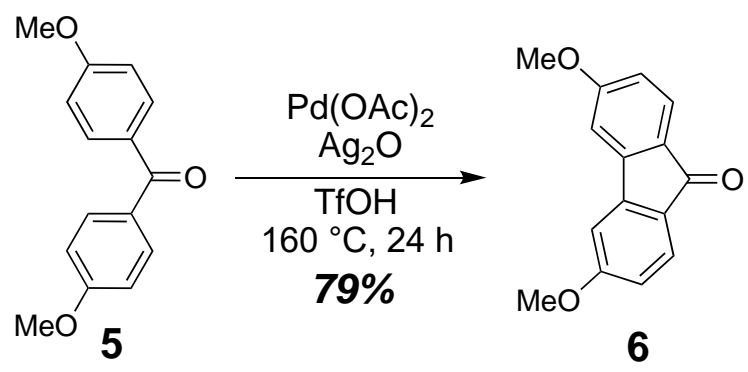

6

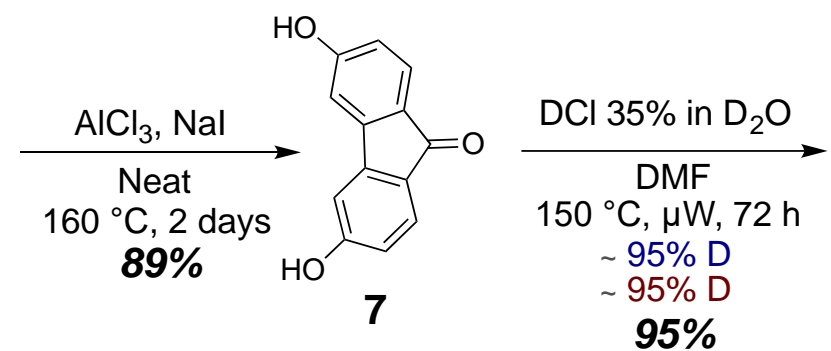

9H-fluorene-2,4,5,7- $d_{4}$<smiles>[2H]c1cc2c(c([2H])c1O)-c1c(cc([2H])c(O)c1[2H])C2=O</smiles>

8<smiles>[2H]c1cc2c(c([2H])c1O)-c1c(cc([2H])c(O)c1[2H])C2=O</smiles>

9

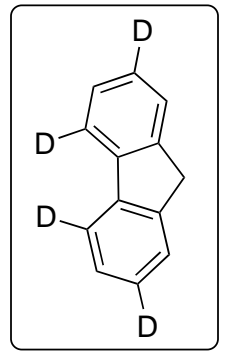

10

Figure S2. General synthetic route for $9 H$-fluorene-2,4,5,7-d

Synthesis of $9 H$-fluorene-1,3,6,8- $d_{4}$

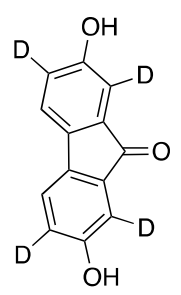




\section{2,7-dihydroxy-9H-fluoren-9-one-1,3,6,8- $d_{4}(2)$}

In a $20 \mathrm{~mL}$ microwave reaction vial with a magnetic stirrer bar, 2,7-di(hydroxy- $d$ )-9H-fluoren-9one $\left(600 \mathrm{mg}, 2.83 \mathrm{mmol}\right.$ ) was added, followed by $35 \mathrm{wt} . \% \mathrm{DCl}$ solution in $\mathrm{D}_{2} \mathrm{O}(5 \mathrm{~mL})$ and $\mathrm{DMF}$ $(5 \mathrm{~mL})$ under an argon atmosphere. The vial was sealed and heated in the microwave synthesis apparatus for $72 \mathrm{~h}$ at $150{ }^{\circ} \mathrm{C}$. Every $12 \mathrm{~h}$, the mixture was cooled to room temperature, opened to relieve pressure and an additional $3 \mathrm{~mL}$ of $35 \mathrm{wt} . \% \mathrm{DCl}$ solution in $\mathrm{D}_{2} \mathrm{O}$ was added. The reaction was suggested 6 times to microwave irradiation at $150^{\circ} \mathrm{C}$ for $12 \mathrm{~h}$ each to reach a higher deuterium incorporation. Reaction follow-up was done by ${ }^{1} \mathrm{H}-\mathrm{NMR}$. Then, the mixture was cooled to room temperature, and was added to ice-cold $\mathrm{H}_{2} \mathrm{O}(20 \mathrm{~mL})$, forming a reddish precipitate. The precipitate was filtered on a fritted funnel and washed with hexanes $(3 \times 10 \mathrm{~mL})$, saturated aqueous sodium bicarbonate solution $(3 \times 20 \mathrm{~mL})$ and water $(3 \times 20 \mathrm{~mL})$. The solid residue was dried over $\mathrm{MgSO}_{4}$ and then dried overnight in a vacuum oven at $100^{\circ} \mathrm{C}$ to yield 2,7-dihydroxy-9H-fluoren-9-one$1,3,6,8-d_{4}$ as a red solid $(580 \mathrm{mg}, 95 \%)$. The total incorporation yield for both positions was determined by ${ }^{1} \mathrm{H}$ NMR spectroscopy relative to the intensity of a nonexchangeable proton in the molecule, and was further confirmed by LC-MS analysis. Deuterium incorporation (Position 1 and 8: 93\%; Position 3 and 6: 93\%).

DP:

${ }^{1} \mathrm{H}$ NMR $\left(600 \mathrm{MHz}, \mathrm{DMSO}-d_{6}\right) \delta 9.83(\mathrm{~s}, 2 \mathrm{H}), 7.37$ (s, 2H).

${ }^{13} \mathrm{C}$ NMR (151 MHz, DMSO-d $) \delta 193.40,157.47,135.68,135.06,120.96,120.70(\mathrm{~m}), 110.83$ (m).

HRMS (DART/AccuTOF) m/z: [M] $]^{+}$Calcd for $\mathrm{C}_{13} \mathrm{H}_{4} \mathrm{D}_{4} \mathrm{O}_{3}$ 217.0758; Found 217.0769.

NDI:

${ }^{1} \mathrm{H}$ NMR (600 MHz, DMSO-d $\left.d_{6}\right) \delta 6.88(\mathrm{~d}, J=2.6 \mathrm{~Hz}, 0.14 \mathrm{H}), 6.86(\mathrm{dd}, J=8.0,2.3 \mathrm{~Hz}, 0.14 \mathrm{H})$.

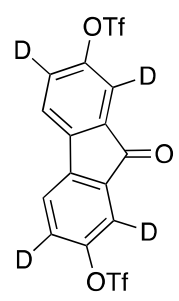

\section{9-oxo-9H-fluorene-2,7-diyl-1,3,6,8- $\boldsymbol{d}_{4}$ bis(trifluoromethanesulfonate) (3)}

To a solution of 2,7-di(hydroxy- $d$ )- $9 H$-fluoren-9-one-1,3,6,8- $d_{4}(550 \mathrm{mg}, 2.54 \mathrm{mmol})$ and pyridine $(1 \mathrm{~mL})$ in dichloromethane $(8 \mathrm{~mL})$ was added a $1 \mathrm{M}$ solution of $\mathrm{Tf}_{2} \mathrm{O}$ in $\mathrm{DCM}(6.35 \mathrm{~mL}, 6.35$ $\mathrm{mmol})$ at $0^{\circ} \mathrm{C}$. The stirring was continued overnight at room temperature before it was poured into water and the organic phase was extracted with dichloromethane $(3 \times 10 \mathrm{~mL})$. The combined organic phases were washed subsequently with $3 \%$ aqueous $\mathrm{HCl}$ solution $(2 \times 10 \mathrm{~mL})$, saturated aqueous sodium bicarbonate solution $(1 \times 10 \mathrm{~mL})$ and brine $(2 \times 10 \mathrm{~mL})$ and then dried over $\mathrm{MgSO}_{4}$. The reaction mixture was concentrated in vacuo and the purple residue was directly purified by flash chromatography on silica gel (hexanes/ethyl acetate, 90:10) to yield

9-oxo-9H-fluorene-2,7-diyl-1,3,6,8- $d_{4}$ bis(trifluoromethanesulfonate) as a purple solid (1.12 $\mathrm{g}$, $92 \%)$.

DP: 
${ }^{1} \mathrm{H}$ NMR $\left(600 \mathrm{MHz}, \mathrm{CDCl}_{3}\right) \delta 7.66(\mathrm{~s}, 2 \mathrm{H})$.

${ }^{13} \mathrm{C} \mathrm{NMR}\left(151 \mathrm{MHz}, \mathrm{CDCl}_{3}\right) \delta 189.26,150.46,142.72,136.32,127.73(\mathrm{t}, J=25.5 \mathrm{~Hz}) 122.41$, $119.89,118.36,118.12(\mathrm{t}, J=25.5 \mathrm{~Hz}), 117.76,115.64$.

HRMS (DART/AccuTOF) m/z: [M] $]^{+}$Calcd for $\mathrm{C}_{15} \mathrm{H}_{2} \mathrm{D}_{4} \mathrm{~F}_{6} \mathrm{O}_{7} \mathrm{~S}_{2} 480.9744$; Found 480.9774.

NDI:

${ }^{1} \mathrm{H}$ NMR $\left(600 \mathrm{MHz}, \mathrm{CDCl}_{3}\right) \delta 7.61(\mathrm{~d}, J=1.8 \mathrm{~Hz}, 0.13 \mathrm{H}), 7.46(\mathrm{dd}, J=8.1,1.9 \mathrm{~Hz}, 0.13 \mathrm{H})$.

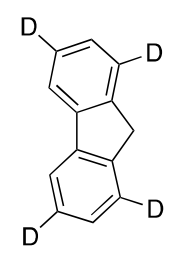

\section{$9 H$-fluorene-1,3,6,8- $d_{4}(4)$}

To a round-bottom flask under an argon atmosphere, $10 \mathrm{wt} . \%$ palladium on carbon (300 mg, 0.28 mmol) was added, then a 1:1 mixture of THF/MeOH $(10 \mathrm{~mL})$ was added slowly and the reaction was degassed with argon for $20 \mathrm{~min}$. Then, 9-oxo-9H-fluorene-2,7-diyl-1,3,6,8- $d_{4}$ bis(trifluoromethanesulfonate) $(1.0 \mathrm{~g}, 2.08 \mathrm{mmol})$ was added and the reaction was bubbled with 2 atmosphere of hydrogen gas for $20 \mathrm{~min}$. The reaction was sealed and left to stir for $24 \mathrm{~h}$ at $50^{\circ} \mathrm{C}$ or until complete consumption of starting material. The reaction was followed by TLC using fluorene as co-spot. The reaction was then filtered on Celite and the reaction mixture was concentrated in vacuo to afford a beige residue. Further purification by flash chromatography on silica gel (hexanes/ethyl acetate, 95:5) yielded $9 H$-fluorene-1,3,6,8- $d_{4}$ as a white solid (184 mg, $52 \%$ ).

\section{DP:}

${ }^{1} \mathrm{H}$ NMR (600 MHz, $\left.\mathrm{CDCl}_{3}\right) \delta 7.82(\mathrm{~s}, 2 \mathrm{H}), 7.33(\mathrm{~s}, 2 \mathrm{H}), 3.93(\mathrm{~s}, 2 \mathrm{H})$.

${ }^{13} \mathrm{C} \mathrm{NMR}\left(151 \mathrm{MHz}, \mathrm{CDCl}_{3}\right) \delta$ 143.26, 141.84, 126.61, 126.56 (t, $\left.24.5 \mathrm{~Hz}\right), 124.86$ (t, $\left.24.5 \mathrm{~Hz}\right)$, 119.89, 36.97.

HRMS (DART/AccuTOF) m/z: [M] ${ }^{+}$Calcd for $\mathrm{C}_{13} \mathrm{H}_{6} \mathrm{D}_{4}$ 171.1067; Found 171.0933.

\section{NDI:}

${ }^{1} \mathrm{H}$ NMR $\left(600 \mathrm{MHz}, \mathrm{CDCl}_{3}\right) \delta 7.57(\mathrm{dd}, J=7.4,1.0 \mathrm{~Hz}, 0.14 \mathrm{H}), 7.40(\mathrm{t}, J=7.5 \mathrm{~Hz}, 0.14 \mathrm{H})$.

\section{Synthesis of $9 H$-fluorene-2,4,5,7-d4}

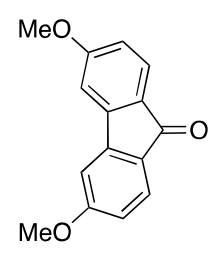

\section{3,6-dimethoxy-9H-fluoren-9-one (6)}

Synthesized following a modified literature procedure[1]. In a microwave reaction vial with a magnetic stirrer bar, 4,4'-dimethoxybenzophenone (5.0 g, $20.6 \mathrm{mmol}$ ), palladium(II) acetate (463 
mg, $2.06 \mathrm{mmol}$ ), and silver(I) oxide (7.05 g, $30.45 \mathrm{mmol})$ was added, followed by trifluoroacetic acid $(15 \mathrm{~mL})$ under an argon atmosphere. The vial was sealed and heated in the microwave synthesis apparatus for $12 \mathrm{~h}$ at $160{ }^{\circ} \mathrm{C}$. After completion of the reaction, the reaction mixture was cooled to room temperature, filtered through a short Celite pad and was washed several times with dichloromethane. The combined filtrate was concentrated in vacuo and purified by flash chromatography on silica gel (hexanes/ethyl acetate, 70:30) to yield 3,6-dimethoxy-9H-fluoren-9one as a pale-yellow solid (3.92 $\mathrm{g}, 79 \%)$.

${ }^{1} \mathrm{H}$ NMR $\left(600 \mathrm{MHz}, \mathrm{CDCl}_{3}\right) \delta 7.58(\mathrm{~d}, J=8.2 \mathrm{~Hz}, 2 \mathrm{H}), 7.00(\mathrm{~d}, J=2.2 \mathrm{~Hz}, 2 \mathrm{H}), 6.75(\mathrm{dd}, J=8.2$, $2.2 \mathrm{~Hz}, 2 \mathrm{H}), 3.90(\mathrm{~s}, 6 \mathrm{H})$.

\section{3,6-dihydroxy-9H-fluoren-9-one (7)}

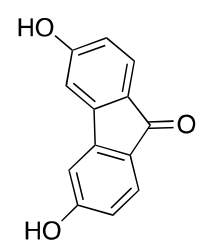

Synthesized following a modified literature procedure[2]. In an unsealed $50 \mathrm{~mL}$ pressure vial, 3,6dimethoxy-9H-fluoren-9-one $(2.0 \mathrm{~g}, 8.32 \mathrm{mmol}), \mathrm{AlCl}_{3}(3.33 \mathrm{~g}, 24.97 \mathrm{mmol})$ and $\mathrm{NaI}(6.24 \mathrm{~g}$, $41.6 \mathrm{mmol}$ ) were heated at $160{ }^{\circ} \mathrm{C}$ for $12 \mathrm{~h}$ under neat conditions. The crude was cooled to room temperature and deionized $\mathrm{H}_{2} \mathrm{O}(50 \mathrm{~mL})$ was added. The mixture was extracted with $3 \times 20 \mathrm{~mL}$ of ethyl acetate and the organic phase was dried over $\mathrm{MgSO}_{4}$, concentrated in vacuo and purified by flash chromatography on silica gel (dichloromethane/ethyl acetate, 95:5) to yield 3,6-dihydroxy9H-fluoren-9-one as a white solid (1.57 g, 89\%).

${ }^{1} \mathrm{H}$ NMR (600 MHz, Acetone- $\left.d_{6}\right) \delta 9.40(\mathrm{~s}, 2 \mathrm{H}), 7.42(\mathrm{~d}, J=8.1 \mathrm{~Hz}, 2 \mathrm{H}), 7.18(\mathrm{~d}, J=2.3 \mathrm{~Hz}, 2 \mathrm{H})$, $6.81(\mathrm{dd}, J=8.1,2.2 \mathrm{~Hz}, 2 \mathrm{H})$.

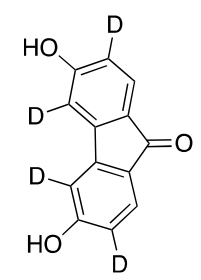

3,6-dihydroxy-9H-fluoren-9-one-2,4,5,7- $d_{4}(8)$

In a $20 \mathrm{~mL}$ microwave reaction vial with a magnetic stirrer bar, 3,6-di(hydroxy- $d$ )-9H-fluoren-9one $\left(600 \mathrm{mg}, 2.83 \mathrm{mmol}\right.$ ) was added, followed by $35 \mathrm{wt} . \% \mathrm{DCl}$ solution in $\mathrm{D}_{2} \mathrm{O}(5 \mathrm{~mL})$ and DMF $(5 \mathrm{~mL})$ under an argon atmosphere. The vial was sealed and heated in the microwave synthesis apparatus for $72 \mathrm{~h}$ at $150{ }^{\circ} \mathrm{C}$. Every $12 \mathrm{~h}$, the mixture was cooled to room temperature, opened to relieve pressure and an additional $3 \mathrm{~mL}$ of $35 \mathrm{wt} . \% \mathrm{DCl}$ solution in $\mathrm{D}_{2} \mathrm{O}$ was added. The reaction was suggested 6 times to microwave irradiation at $150^{\circ} \mathrm{C}$ for $12 \mathrm{~h}$ each to reach a higher deuterium incorporation. The mixture was cooled to room temperature and was added to ice-cold $\mathrm{H}_{2} \mathrm{O}$ (20 $\mathrm{mL}$ ), forming a yellow precipitate. The precipitate was filtered on a fritted funnel and, washed with hexanes $(3 \times 10 \mathrm{~mL})$, saturated aqueous sodium bicarbonate solution $(1 \times 10 \mathrm{~mL})$ and water $(3 \times 20 \mathrm{~mL})$. The solid residue was dried overnight in a vacuum oven at $100^{\circ} \mathrm{C}$ to yield $3,6-$ dihydroxy-9H-fluoren-9-one-2,4,5,7- $d_{4}$ as a yellow solid (580 mg, 95\%). The total incorporation 
yield for both positions was determined by ${ }^{1} \mathrm{H}$ NMR spectroscopy relative to the intensity of a nonexchangeable proton in the molecule, and was further confirmed by LC-MS analysis. Deuterium incorporation (Position 2 and 7: 95\%; Position 4 and 5: 95\%)

\section{DP:}

${ }^{1} \mathrm{H}$ NMR (600 MHz, DMSO-d $\left.d_{6}\right) \delta 10.51$ (s, 2H), 7.38 (s, 2H).

${ }^{13} \mathrm{C}$ NMR $\left(151 \mathrm{MHz}, \mathrm{DMSO}-d_{6}\right) \delta 190.31,163.59,145.70,126.01,125.33,114.72(\mathrm{~m}), 108.27$

(t, $23.5 \mathrm{~Hz})$

HRMS (DART/AccuTOF) m/z: [M] ${ }^{+}$Calcd for $\mathrm{C}_{13} \mathrm{H}_{4} \mathrm{D}_{4} \mathrm{O}_{3} 217.0758$; Found 217.0742.

\section{NDI:}

${ }^{1} \mathrm{H}$ NMR (600 MHz, DMSO-d $) \delta 7.04(\mathrm{~s}, 0.09 \mathrm{H}), 6.67(\mathrm{~d}, J=8.1 \mathrm{~Hz}, 0.10 \mathrm{H})$.

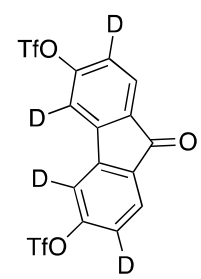

\section{9-oxo-9H-fluorene-3,6-diyl-2,4,5,7- $d_{4}$ bis(trifluoromethanesulfonate) (9)}

Prepared according to the general procedure used for compound 2, 2,7-di(hydroxy- $d$ )-9H-fluoren9-one-1,3,6,8- $d_{4}(500 \mathrm{mg}, 2.36 \mathrm{mmol})$, pyridine $(1 \mathrm{~mL})$, dichloromethane $(7.5 \mathrm{~mL})$, and $1 \mathrm{M}$ solution of $\mathrm{Tf}_{2} \mathrm{O}$ in DCM $(5.90 \mathrm{~mL}, 5.90 \mathrm{mmol})$ were used. Yielding 9-oxo-9H-fluorene-3,6-diyl2,4,5,7- $d_{4}$ bis(trifluoromethanesulfonate) as a yellow solid (1.07 $\mathrm{g}, 88 \%$ ).

\section{DP:}

${ }^{1} \mathrm{H}$ NMR $\left(600 \mathrm{MHz}, \mathrm{CDCl}_{3}\right) \delta 7.81(\mathrm{~s}, 2 \mathrm{H})$.

${ }^{13} \mathrm{C} \mathrm{NMR}\left(151 \mathrm{MHz}, \mathrm{CDCl}_{3}\right) \delta 189.47,154.02,145.01,133.67,126.57,122.87$ (t, $\left.25.0 \mathrm{~Hz}\right)$, $119.88,117.76,114.68(\mathrm{t}, 25.0 \mathrm{~Hz})$.

HRMS (DART/AccuTOF) m/z: [M] $]^{+}$Calcd for $\mathrm{C}_{15} \mathrm{H}_{2} \mathrm{D}_{4} \mathrm{~F}_{6} \mathrm{O}_{7} \mathrm{~S}_{2} 480.9844$; Found 480.9842.

\section{NDI:}

${ }^{1} \mathrm{H}$ NMR $\left(600 \mathrm{MHz}^{\mathrm{CDCl}} 3\right) \delta 7.48(\mathrm{~s}, 0.08 \mathrm{H}), 7.30(\mathrm{~d}, J=8.1 \mathrm{~Hz}, 0.09 \mathrm{H})$.

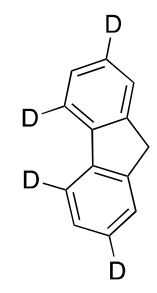

\section{H-fluorene-2,4,5,7- $d_{4}(\mathbf{1 0})$}

Prepared according to the general procedure used for compound 3,10 wt.\% palladium on carbon (240 mg, $0.22 \mathrm{mmol}$ ), 1:1 mixture of THF/MeOH (8 mL), 9-oxo-9H-fluorene-2,7-diyl-1,3,6,8- $d_{4}$ 
bis(trifluoromethanesulfonate) (800 mg, $1.66 \mathrm{mmol}$ ) was used. Yielding 9H-fluorene-2,4,5,7- $d_{4}$ as a white solid (170 mg, 60\%).

DP:

${ }^{1} \mathrm{H}$ NMR $\left(600 \mathrm{MHz}, \mathrm{CDCl}_{3}\right) \delta 7.56(\mathrm{~s}, 1 \mathrm{H}), 7.39(\mathrm{~s}, 1 \mathrm{H}), 3.92(\mathrm{~s}, 1 \mathrm{H})$.

${ }^{13} \mathrm{C} \mathrm{NMR}\left(151 \mathrm{MHz}, \mathrm{CDCl}_{3}\right) \delta 143.34,141.75,126.68,126.53(\mathrm{t}, 24.5 \mathrm{~Hz}), 125.05,119.74(\mathrm{t}, 24.5$ $\mathrm{Hz}), 37.06$.

HRMS (DART/AccuTOF) m/z: [M] $]^{+}$Calcd for $\mathrm{C}_{13} \mathrm{H}_{6} \mathrm{D}_{4}$ 171.1067; Found 171.0934.

NDI:

${ }^{1} \mathrm{H}$ NMR $\left(600 \mathrm{MHz}, \mathrm{CDCl}_{3}\right) \delta 7.81(\mathrm{~d}, J=7.6 \mathrm{~Hz}, 0.10 \mathrm{H}), 7.32(\mathrm{t}, J=7.4 \mathrm{~Hz}, 0.11 \mathrm{H})$.

\section{Synthesis of Selectively Deuterated and Perdeuterated BDPA Radicals}
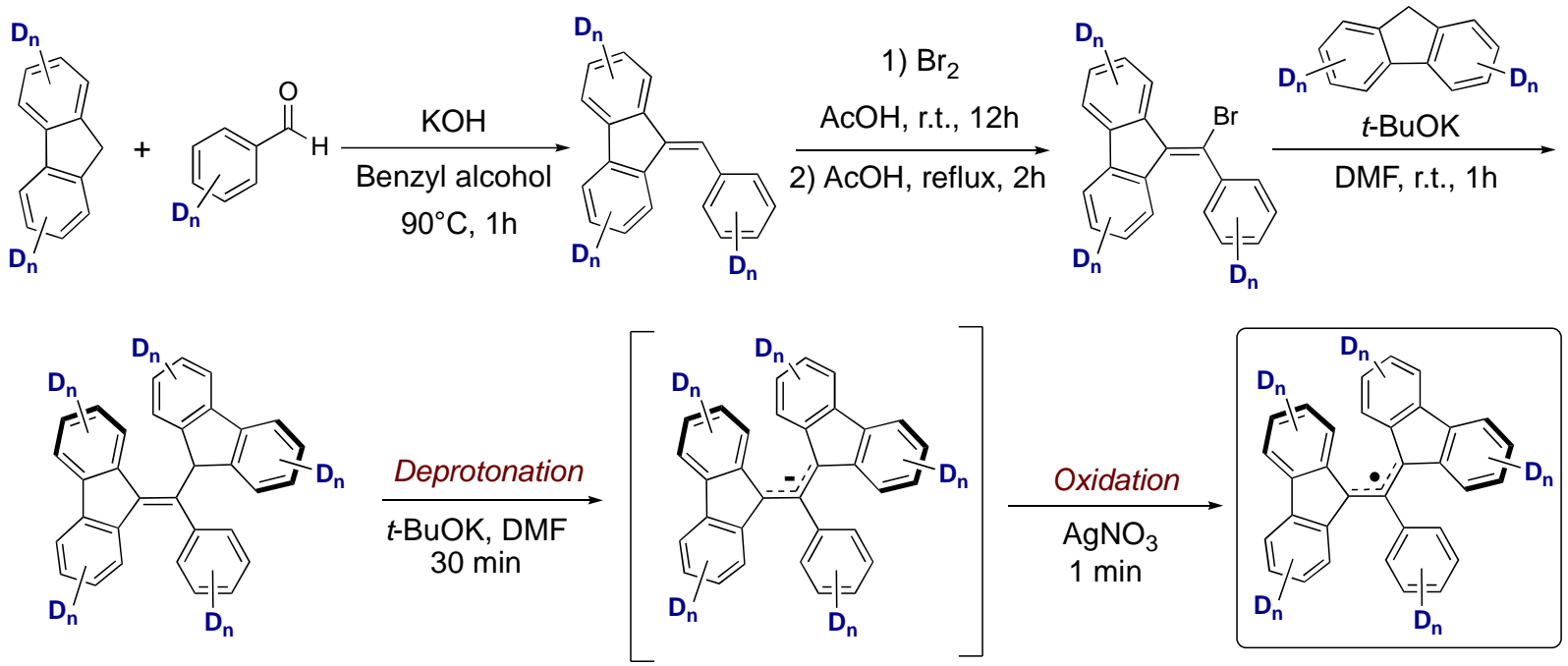

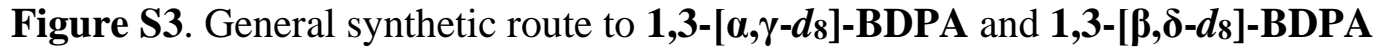

\section{Synthesis of 1,3-[ $\alpha, \gamma-d 8]-B D P A$ radical}

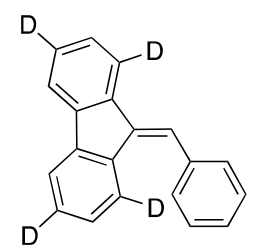

\section{9-benzylidene-9H-fluorene-1,3,6,8- $d_{4}(11)$}

Synthesized following a modified literature procedure[3]. Benzaldehyde $(60 \mu \mathrm{L}, 0.587 \mathrm{mmol})$ was added dropwise to a solution of $9 \mathrm{H}$-fluorene-1,3,6,8- $d_{4}(100 \mathrm{mg}, 0.587 \mathrm{mmol})$ and $\mathrm{CsOH} \cdot \mathrm{H}_{2} \mathrm{O}(40$ 
$\mathrm{mg}, 0.235 \mathrm{mmol})$ in $\mathrm{EtOH}(1.2 \mathrm{~mL})$ and the mixture was stirred at $25{ }^{\circ} \mathrm{C}$ for $2 \mathrm{~h}$. Complete consumption of the starting materials was observed by TLC and the reaction mixture was concentrated in vacuo. The crude reaction mixture was purified by flash chromatography on silica gel (hexanes/ethyl acetate, 80:20) to yield 9-benzylidene-9H-fluorene-1,3,6,8- $d_{4}$ as a pale-yellow solid (131 mg, 86\%).

\section{DP:}

${ }^{1} \mathrm{H} \mathrm{NMR}\left(600 \mathrm{MHz}, \mathrm{CDCl}_{3}\right) \delta 7.77-7.69(\mathrm{~m}, 3 \mathrm{H}), 7.60(\mathrm{~d}, J=7.2 \mathrm{~Hz}, 2 \mathrm{H}), 7.47(\mathrm{t}, J=7.6 \mathrm{~Hz}$, $2 \mathrm{H}), 7.40(\mathrm{t}, J=7.6 \mathrm{~Hz}, 1 \mathrm{H}), 7.34(\mathrm{~s}, 1 \mathrm{H}), 7.06(\mathrm{~s}, 1 \mathrm{H})$.

${ }^{13} \mathrm{C}$ NMR $\left(151 \mathrm{MHz}, \mathrm{CDCl}_{3}\right) \delta 146.88,141.39,139.56,139.33,137.04,136.60,129.40,128.67$, 128.17, 127.42, 126.91, 126.58, 124.99 - 124.07 (m), 120.39 - 119.79 (m), 119.74, 119.61.

HRMS (DART/AccuTOF) m/z: [M] ${ }^{+}$Calcd for $\mathrm{C}_{20} \mathrm{H}_{10} \mathrm{D}_{4} 259.1180$; Found 259.1158.

\section{NDI:}

${ }^{1} \mathrm{H}$ NMR $\left(600 \mathrm{MHz}, \mathrm{CDCl}_{3}\right) \delta 7.80(\mathrm{~d}, J=7.6 \mathrm{~Hz}, 0.16 \mathrm{H}), 7.56(\mathrm{~d}, J=7.8 \mathrm{~Hz}, 0.16 \mathrm{H}), 7.31(\mathrm{~d}, J$ $=7.4 \mathrm{~Hz}, 0.23 \mathrm{H}), 7.25-7.21(\mathrm{~m}, 0.48 \mathrm{H})$.

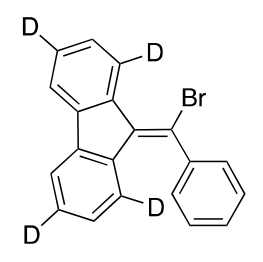

\section{9-(bromo(phenyl)methylene)-9H-fluorene-1,3,6,8- $d_{4}$ (12)}

Synthesized following a modified literature procedure[3]. To a flask covered with aluminum foil was suspended 9-benzylidene-9H-fluorene-1,3,6,8- $d_{4}(70 \mathrm{mg}, 0.27 \mathrm{mmol})$ in acetic acid $(1 \mathrm{~mL})$. The reaction was cooled to $0^{\circ} \mathrm{C}$, then $\mathrm{Br}_{2}(13 \mu \mathrm{L}, 0.26 \mathrm{mmol})$ was added dropwise while stirring heavily. The reaction mixture was warmed at room temperature and stir overnight. Then, $50 \mathrm{~mL}$ of water was added to precipitate the product. The mixture was extracted with dichloromethane (3 $\mathrm{x} 5 \mathrm{~mL}$ ) and the organic phases were washed with saturated aqueous sodium bicarbonate solution ( 3 x $20 \mathrm{~mL}$ ) and water $(3 \times 20 \mathrm{~mL})$. The organic phase was concentrated in vacuo and the intermediate product 9-bromo-9-[bromo(phenyl)methyl]-9H-fluorene was dried for $2 \mathrm{~h}$ before being suspended in acetic acid $(2 \mathrm{ml})$ and heated at reflux for $2 \mathrm{~h}$. The reaction mixture was left to cool at room temperature overnight and the product was collected by filtration and dried under vacuum to give the crude desired product with a $90 \%$ purity. The crude mixture was further purified by flash chromatography on silica gel (hexanes/ethyl acetate, 85:15) to yield 9(bromo(phenyl)methylene)-9H-fluorene-1,3,6,8- $d_{4}$ as a yellow solid (54 $\mathrm{mg}, 59 \%$ ).

\section{DP:}

${ }^{1} \mathrm{H} \mathrm{NMR}\left(600 \mathrm{MHz}, \mathrm{CDCl}_{3}\right) \delta 7.74(\mathrm{~s}, 1 \mathrm{H}), 7.65$ (s, 1H), $7.56-7.44$ (m, 5H), $7.40(\mathrm{~s}, 1 \mathrm{H}), 6.85$ (s, $1 \mathrm{H})$.

${ }^{13} \mathrm{C}$ NMR $\left(151 \mathrm{MHz}, \mathrm{CDCl}_{3}\right) \delta 142.96,141.35,139.95,138.24,137.99,136.17,129.40,129.38$, $129.30-128.73(\mathrm{~m}), 128.69,128.55-127.76(\mathrm{~m}), 127.01,126.75,126.20-125.67(\mathrm{~m}), 124.8-$ $124.35(\mathrm{~m}), 121.04-120.38(\mathrm{~m}), 119.56,119.28$.

HRMS (DART/AccuTOF) m/z: [M] ${ }^{+}$Calcd for $\mathrm{C}_{20} \mathrm{H}_{9} \mathrm{D}_{4} \mathrm{Br} 337.0412$; Found 337.0382. 


\section{NDI:}

${ }^{1} \mathrm{H}$ NMR $\left(600 \mathrm{MHz}, \mathrm{CDCl}_{3}\right) \delta 8.88(\mathrm{~d}, J=8.0 \mathrm{~Hz}, 0.07 \mathrm{H}), 7.70(\mathrm{~s}, 0.08 \mathrm{H}), 7.62-7.58(\mathrm{~m}$, $0.17 \mathrm{H}), 7.34(\mathrm{~s}, 0.07 \mathrm{H}), 7.23(\mathrm{t}, J=7.5 \mathrm{~Hz}, 0.08 \mathrm{H}), 7.06(\mathrm{~s}, 0.06 \mathrm{H}), 6.21(\mathrm{~d}, J=8.0 \mathrm{~Hz}, 0.07 \mathrm{H})$.

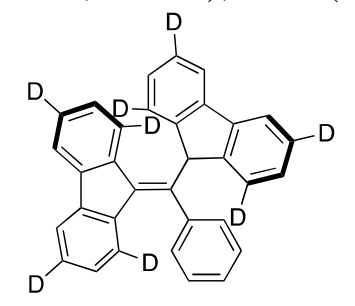

\section{9-((9H-fluoren-9-yl-1,3,6,8- $\left.d_{4}\right)\left(\right.$ phenyl)methylene)-9H-fluorene-1,3,6,8- $d_{4}(13)$}

Synthesized following a modified literature procedure[3]. ${ }^{\mathrm{B}} \mathrm{BuOK}(27 \mathrm{mg}, 0.24 \mathrm{mmol})$ was added in portions to a stirred solution of 9-(bromo(phenyl)methylene)-9H-fluorene-1,3,6,8- $d_{4}$ (20 mg, $0.059 \mathrm{mmol})$ and $9 H$-fluorene-1,3,6,8- $d_{4}(11 \mathrm{mg}, 0.065 \mathrm{mmol})$ in DMF $(0.5 \mathrm{~mL})$ under $\mathrm{N}_{2}$. The reaction mixture was stirred $3 \mathrm{~h}$ at room temperature, then hydrochloric acid $(1 \mathrm{M}, 1 \mathrm{~mL}$ was added and the reaction mixture followed by water $(2 \mathrm{ml})$ to give an orange precipitate. The crude mixture was collected by filtration, washed with water $(10 \mathrm{ml})$ and methanol $(10 \mathrm{ml})$ and dried overnight. Purification by flash chromatography on silica gel (hexanes/ethyl acetate, 80:20) or recrystallization in benzene gave the 9-((9H-fluoren-9-yl-1,3,6,8- $\left.d_{4}\right)$ (phenyl)methylene)-9Hfluorene-1,3,6,8- $d_{4}$ as an orange solid (20 mg, 78\%).

\section{DP:}

${ }^{1} \mathrm{H}$ NMR $\left(600 \mathrm{MHz}, \mathrm{CDCl}_{3}\right) \delta 7.89(\mathrm{~s}, 1 \mathrm{H}), 7.75(\mathrm{~s}, 1 \mathrm{H}), 7.66(\mathrm{~s}, 2 \mathrm{H}), 7.36(\mathrm{~s}, 1 \mathrm{H}), 7.26(\mathrm{~s}, 2 \mathrm{H})$, $7.07(\mathrm{t}, J=7.4 \mathrm{~Hz}, 1 \mathrm{H}), 6.99(\mathrm{t}, J=7.6 \mathrm{~Hz}, 2 \mathrm{H}), 6.81(\mathrm{~s}, 1 \mathrm{H}), 6.67(\mathrm{~d}, J=7.1 \mathrm{~Hz}, 2 \mathrm{H}), 6.48$ (s, $1 \mathrm{H})$.

${ }^{13} \mathrm{C}$ NMR $\left(151 \mathrm{MHz}, \mathrm{CDCl}_{3}\right) \delta 145.27,144.22,142.11,141.44,139.89,139.00,138.78,138.71$, 136.06, 128.48, 127.86, 127.36, 127.20, 126.83, 126.48, $126.09-125.63$ (m), $125.21-124.69$ (m), 119.99, 119.95, 119.08, 52.79.

HRMS (DART/AccuTOF) m/z: [M] ${ }^{+}$Calcd for $\mathrm{C}_{33} \mathrm{H}_{14} \mathrm{D}_{8} 427.2257$; Found 427.2345.

\section{NDI:}

${ }^{1} \mathrm{H}$ NMR $\left(600 \mathrm{MHz}, \mathrm{CDCl}_{3}\right) \delta 8.44(\mathrm{~d}, J=8.0 \mathrm{~Hz}, 0.07 \mathrm{H}), 7.60(\mathrm{~d}, J=7.6 \mathrm{~Hz}, 0.14 \mathrm{H}), 7.47(\mathrm{t}, J$ $=7.5 \mathrm{~Hz}, 0.08 \mathrm{H}), 7.34(\mathrm{t}, J=7.6 \mathrm{~Hz}, 0.14 \mathrm{H}), 7.22(\mathrm{t}, J=7.3 \mathrm{~Hz}, 0.15 \mathrm{H}), 6.49(\mathrm{~s}, 0.08 \mathrm{H}), 5.90(\mathrm{~d}$, $J=8.0 \mathrm{~Hz}, 0.07 \mathrm{H})$.

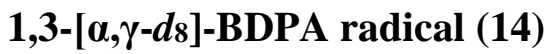

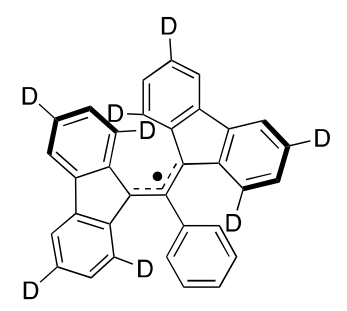


Synthesized following a modified literature procedure[4]. ${ }^{\mathrm{B}} \mathrm{BuOK}(24 \mathrm{mg}, 0.21 \mathrm{mmol})$ was added in portions to a stirred solution of compound 9-( $9 H$-fluoren-9-yl-1,3,6,8- $\left.d_{4}\right)$ (phenyl)methylene)$9 H$-fluorene-1,3,6,8- $d_{4}(15 \mathrm{mg}, 0.035 \mathrm{mmol})$ in DMF $(0.3 \mathrm{ml})$ under $\mathrm{N}_{2}$ at room temperature which resulted in a colour change from orange to dark blue. After $30 \mathrm{~min}$, a solution of $\mathrm{AgNO}_{3}(35 \mathrm{mg}$, $0.21 \mathrm{mmol})$ in DMF $(0.1 \mathrm{~mL})$ was added. The solution immediately became red-brown and was diluted after $30 \mathrm{~min}$ of stirring with $2 \mathrm{~mL}$ of $0.01 \mathrm{M} \mathrm{HCl}$ and extracted with diethyl ether (3x2 $\mathrm{mL})$. The combined organic layers were washed with water $(3 \times 10 \mathrm{~mL})$ and dried under vacuum. Purification by flash chromatography on silica gel (hexanes/ethyl acetate, 80:20) gave the 1,3-[ $\alpha, \gamma-$ $\left.d_{8}\right]$-BDPA radical as a deep red glassy solid (10 $\left.\mathrm{mg}, 66 \%\right)$.

\section{Synthesis of 1,3-[ $[\beta, \delta-d 8]-B D P A$ radical}

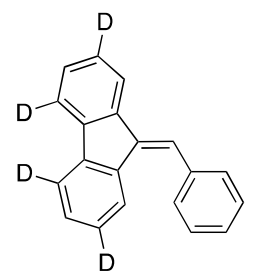

\section{9-benzylidene-9H-fluorene-2,4,5,7-d $(15)$}

Prepared according to the general procedure used for compound 11. Benzaldehyde $(60 \mu \mathrm{L}, 0.587$ mmol), $9 H$-fluorene-2,4,5,7- $d_{4}(100 \mathrm{mg}, 0.587 \mathrm{mmol})$ and $\mathrm{CsOH} \cdot \mathrm{H}_{2} \mathrm{O}(40 \mathrm{mg}, 0.235 \mathrm{mmol})$, and $\mathrm{EtOH}(1.2 \mathrm{~mL})$ was used. Yielding 9-benzylidene-9H-fluorene-2,4,5,7- $d_{4}$ as a pale-yellow solid (136 mg, 90\%).

\section{DP:}

${ }^{1} \mathrm{H} \mathrm{NMR}\left(600 \mathrm{MHz}, \mathrm{CDCl}_{3}\right) \delta 7.80(\mathrm{~s}, 1 \mathrm{H}), 7.71(\mathrm{~s}, 1 \mathrm{H}), 7.59(\mathrm{~d}, J=8.1 \mathrm{~Hz}, 2 \mathrm{H}), 7.56(\mathrm{~s}, 1 \mathrm{H})$, $7.47(\mathrm{t}, J=7.5 \mathrm{~Hz}, 2 \mathrm{H}), 7.43-7.36(\mathrm{~m}, 2 \mathrm{H}), 7.31(\mathrm{~s}, 1 \mathrm{H})$.

${ }^{13} \mathrm{C}$ NMR $\left(151 \mathrm{MHz}, \mathrm{CDCl}_{3}\right) \delta 141.29,139.61,139.24,137.04,136.67,136.62,129.40,128.67$, 128.46, 128.16, 128.13, 127.40, 127.14 - 126.30 (m), 124.44, 120.26, 119.90 - 119.25 (m).

HRMS (DART/AccuTOF) m/z: [M] ${ }^{+}$Calcd for $\mathrm{C}_{20} \mathrm{H}_{10} \mathrm{D}_{4} 259.1180$; Found 259.1168 .

\section{NDI:}

${ }^{1} \mathrm{H}$ NMR $\left(600 \mathrm{MHz}, \mathrm{CDCl}_{3}\right) \delta 7.75-7.71(\mathrm{~m}, 0.06 \mathrm{H}), 7.35-7.32(\mathrm{~m}, 0.06 \mathrm{H}), 7.06(\mathrm{td}, J=7.6$, $1.3 \mathrm{~Hz}, 0.06 \mathrm{H})$.

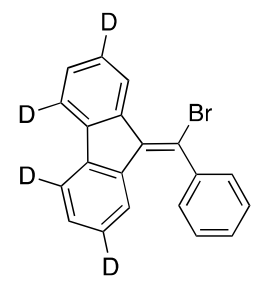

9-(bromo(phenyl)methylene)-9H-fluorene-2,4,5,7-d4 (16) 
Prepared according to the general procedure used for compound 12. 9-Benzylidene-9H-fluorene2,4,5,7-d $d_{4}(70 \mathrm{mg}, 0.27 \mathrm{mmol}), \mathrm{Br}_{2}(13 \mu \mathrm{L}, 0.26 \mathrm{mmol})$ and acetic acid $(1+2 \mathrm{~mL})$ was used. Yielding 9-(bromo(phenyl)methylene)-9H-fluorene-2,4,5,7-d as a pale-yellow solid (49 $\mathrm{mg}$, $54 \%)$.

DP:

${ }^{1} \mathrm{H}$ NMR $\left(600 \mathrm{MHz}, \mathrm{CDCl}_{3}\right) \delta 8.87(\mathrm{~s}, 1 \mathrm{H}), 7.60-7.38(\mathrm{~m}, 6 \mathrm{H}), 7.23(\mathrm{~s}, 1 \mathrm{H}), 6.21(\mathrm{~s}, 1 \mathrm{H})$.

${ }^{13} \mathrm{C}$ NMR $\left(151 \mathrm{MHz}, \mathrm{CDCl}_{3}\right) \delta 142.98,141.27,139.87,138.32,138.07,136.20,129.40,129.39$, $129.06,128.70,128.05,127.25$ - 126.46 (m), 126.08, 124.76, 124.65, 124.44, 120.26, 119.71 $118.91(\mathrm{~m})$.

HRMS (DART/AccuTOF) m/z: [M] ${ }^{+}$Calcd for $\mathrm{C}_{20} \mathrm{H}_{9} \mathrm{D}_{4} \mathrm{Br} 337.0412$; Found 337.0399.

\section{NDI:}

${ }^{1} \mathrm{H}$ NMR $\left(600 \mathrm{MHz}, \mathrm{CDCl}_{3}\right) \delta 7.80(\mathrm{~s}, 0.12 \mathrm{H}), 7.74(\mathrm{~d}, J=7.5 \mathrm{~Hz}, 0.06 \mathrm{H}), 7.71(\mathrm{~s}, 0.11 \mathrm{H}), 7.65$ $(\mathrm{d}, J=7.5 \mathrm{~Hz}, 0.06 \mathrm{H}), 7.56(\mathrm{~s}, 0.07 \mathrm{H}), 7.41(\mathrm{~s}, 0.06 \mathrm{H}) .7 .31(\mathrm{~s}, 0.06 \mathrm{H}), 6.85(\mathrm{t}, J=7.7 \mathrm{~Hz}, 0.07 \mathrm{H})$.

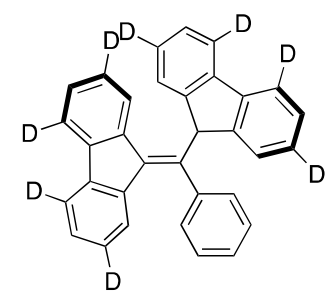

\section{9-((9H-fluoren-9-yl-2,4,5,7-d4)(phenyl)methylene)-9H-fluorene-2,4,5,7-d4 (17)}

Prepared according to the general procedure used for compound 13. ${ }^{\mathrm{t}} \mathrm{BuOK}(27 \mathrm{mg}, 0.24 \mathrm{mmol})$, 9-(bromo(phenyl)methylene)-9H-fluorene-2,4,5,7- $d_{4}$ (20 mg, $\left.0.059 \mathrm{mmol}\right), 9 H$-fluorene-1,3,6,8$d_{4}(11 \mathrm{mg}, 0.065 \mathrm{mmol})$, and DMF $(0.5 \mathrm{ml})$ was used. Yielding 9-( $(9 H$-fluoren-9-yl-2,4,5,7$d_{4}$ )(phenyl)methylene)-9H-fluorene-2,4,5,7- $d_{4}$ as an orange solid (17 $\left.\mathrm{mg}, 68 \%\right)$.

\section{DP:}

${ }^{1} \mathrm{H}$ NMR $\left(600 \mathrm{MHz}, \mathrm{CDCl}_{3}\right) \delta 8.45(\mathrm{~s}, 1 \mathrm{H}), 7.60(\mathrm{~s}, 2 \mathrm{H}), 7.47(\mathrm{~s}, 1 \mathrm{H}), 7.34(\mathrm{~s}, 2 \mathrm{H}), 7.23(\mathrm{~s}, 1 \mathrm{H})$, $7.08(\mathrm{t}, J=7.5 \mathrm{~Hz}, 1 \mathrm{H}), 7.00(\mathrm{t}, J=7.7 \mathrm{~Hz}, 2 \mathrm{H}), 6.67(\mathrm{~d}, J=7.5 \mathrm{~Hz}, 2 \mathrm{H}), 6.50(\mathrm{~s}, 1 \mathrm{H}), 5.90(\mathrm{~s}$, $1 \mathrm{H})$.

${ }^{13} \mathrm{C} \mathrm{NMR}\left(151 \mathrm{MHz}, \mathrm{CDCl}_{3}\right) \delta 145.27,144.30,142.03,141.36,139.81,139.07,138.86,138.71$, $136.11,128.50,127.85,127.37,127.36,127.32,126.20$ - $126.92(\mathrm{~m}), 126.04,125.51,125.14$, $119.55-120.09(\mathrm{~m}), 118.66-119.15(\mathrm{~m}), 52.84$.

HRMS (DART/AccuTOF) m/z: [M] ${ }^{+}$Calcd for $\mathrm{C}_{33} \mathrm{H}_{14} \mathrm{D}_{8} 427.2257$; Found 427.2242.

\section{NDI:}

${ }^{1} \mathrm{H}$ NMR $\left(600 \mathrm{MHz}, \mathrm{CDCl}_{3}\right) \delta 7.89(\mathrm{~d}, J=7.6 \mathrm{~Hz}, 0.06 \mathrm{H}), 7.75(\mathrm{~d}, J=7.6 \mathrm{~Hz}, 0.06 \mathrm{H}), 7.66(\mathrm{~d}, J$ $=7.6 \mathrm{~Hz}, 0.12 \mathrm{H}), 7.37(\mathrm{~d}, J=7.7 \mathrm{~Hz}, 0.06 \mathrm{H}), 6.82(\mathrm{t}, J=7.7 \mathrm{~Hz}, 0.10 \mathrm{H})$. 


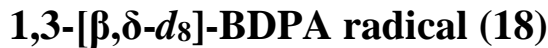

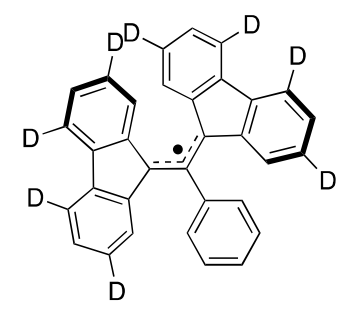

Prepared according to the general procedure used for compound 14. ${ }^{\mathrm{t}} \mathrm{BuOK}(24 \mathrm{mg}, 0.21 \mathrm{mmol})$ was added in portions to a stirred solution of compound 9-( $(9 H$-fluoren-9-yl-2,4,5,7$d_{4}$ )(phenyl)methylene)-9H-fluorene-2,4,5,7-d $(15 \mathrm{mg}, 0.035 \mathrm{mmol}), \mathrm{AgNO}_{3}$ (35 mg, $0.21 \mathrm{mmol}$ ), DMF $(0.3 \mathrm{~mL})$ was used. Yielding $1,3-\left[\beta, \delta-d_{8}\right]$-BDPA radical as a deep red glassy solid (11 $\mathrm{mg}$, $73 \%)$.

\section{Synthesis of 1,3-[ $\left.\alpha, \beta, \gamma, \delta-d_{16}\right]$-BDPA radical}

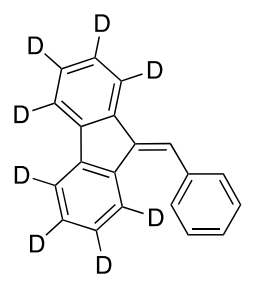

\section{9-benzylidene-9H-fluorene-1,2,3,4,5,6,7,8-ds (19)}

Prepared according to the general procedure used for compound 11. Benzaldehyde ( $461 \mu \mathrm{L}, 4.54$ mmol), $9 \mathrm{H}$-fluorene- $\mathrm{d}_{10}(800 \mathrm{mg}, 4.54 \mathrm{mmol})$ and $\mathrm{CsOH} \cdot \mathrm{H}_{2} \mathrm{O}(305 \mathrm{mg}, 1.81 \mathrm{mmol})$, and EtOH $(9.0 \mathrm{~mL})$ was used. Yielding 9-benzylidene-9H-fluorene-1,2,3,4,5,6,7,8- $d_{8}$ as a pale-yellow solid $(1.05 \mathrm{~g}, 88 \%)$.

\section{DP:}

${ }^{1} \mathrm{H}$ NMR $\left(600 \mathrm{MHz}, \mathrm{CDCl}_{3}\right) \delta 7.73(\mathrm{~s}, 1 \mathrm{H}), 7.63(\mathrm{~d}, J=7.0 \mathrm{~Hz}, 2 \mathrm{H}), 7.50(\mathrm{t}, J=7.4 \mathrm{~Hz}, 2 \mathrm{H})$, $7.43(\mathrm{t}, J=7.2 \mathrm{~Hz}, 1 \mathrm{H})$.

${ }^{13} \mathrm{C}$ NMR $\left(151 \mathrm{MHz}, \mathrm{CDCl}_{3}\right) \delta 141.31,139.55,139.24,137.04,136.60,129.39,128.66,128.14$, $127.38,126.89-125.98(\mathrm{~m}), 124.45-123.80(\mathrm{~m}), 120.30-119.02(\mathrm{~m})$.

HRMS (DART/AccuTOF) m/z: [M] $]^{+}$Calcd for $\mathrm{C}_{20} \mathrm{H}_{6} \mathrm{D}_{8}$ 262.1598; Found 262.1638. 


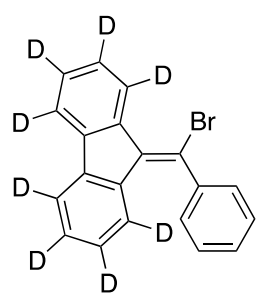

\section{9-(bromo(phenyl)methylene)-9H-fluorene-1,2,3,4,5,6,7,8- $d 8$ (20)}

Prepared according to the general procedure used for compound 12. 9-benzylidene-9H-fluorene$1,2,3,4,5,6,7,8-d_{8}(1.0 \mathrm{~g}, 3.73 \mathrm{mmol}), \mathrm{Br}_{2}(187 \mu \mathrm{L}, 3.65 \mathrm{mmol})$ and acetic acid $(15+15 \mathrm{~mL})$ was used. Yielding 9-(bromo(phenyl)methylene)-9H-fluorene-1,2,3,4,5,6,7,8- $d_{8}$ as a pale-yellow solid (690 mg, 51\%).

${ }^{1} \mathrm{H}$ NMR $\left(600 \mathrm{MHz}, \mathrm{CDCl}_{3}\right) \delta 7.56-7.44(\mathrm{~m}, 5 \mathrm{H})$.

${ }^{13} \mathrm{C} \mathrm{NMR}\left(151 \mathrm{MHz}, \mathrm{CDCl}_{3}\right) \delta 142.97,141.27,139.88,138.24,137.99,136.19,129.39,129.37$, $128.69,127.03-125.24(\mathrm{~m}), 124.62,124.56-124.03(\mathrm{~m}), 119.60-118.70(\mathrm{~m})$.

DP:

HRMS (DART/AccuTOF) m/z: [M] ${ }^{+}$Calcd for $\mathrm{C}_{20} \mathrm{H}_{9} \mathrm{D}_{4} \mathrm{Br} 340.0703$; Found 340.0722.

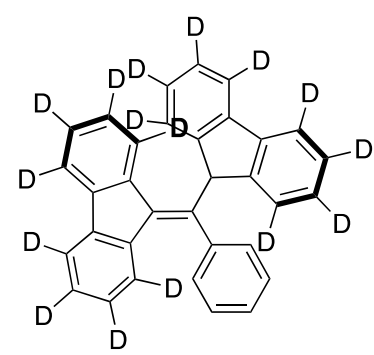

9-((9H-fluoren-9-yl-1,2,3,4,5,6,7,8- $\left.d_{8}\right)\left(\right.$ phenyl)methylene)-9H-fluorene-1,2,3,4,5,6,7,8- $d_{8}(21)$

Prepared according to the general procedure used for compound 13. ${ }^{\mathrm{t}} \mathrm{BuOK}(525 \mathrm{mg}, 04.68 \mathrm{mmol})$, 9-(bromo(phenyl)methylene)-9H-fluorene-1,2,3,4,5,6,7,8- $d_{8}$ (400 mg, $1.17 \mathrm{mmol}$ ), 9H-fluorene$\mathrm{d}_{10}(227 \mathrm{mg}, 1.29 \mathrm{mmol})$, and DMF $(7.3 \mathrm{ml})$ was used. Yielding 9-((9H-fluoren-9-yl$\left.1,2,3,4,5,6,7,8-d_{8}\right)$ (phenyl)methylene)- $9 H$-fluorene-1,2,3,4,5,6,7,8- $d_{8}$ as an orange solid (330 mg, $65 \%)$.

${ }^{1} \mathrm{H}$ NMR $\left(600 \mathrm{MHz}, \mathrm{CDCl}_{3}\right) \delta 7.08(\mathrm{t}, J=7.4 \mathrm{~Hz}, 1 \mathrm{H}), 7.00(\mathrm{t}, J=7.5 \mathrm{~Hz}, 2 \mathrm{H}), 6.68(\mathrm{~d}, J=6.8$ $\mathrm{Hz}, 2 \mathrm{H}), 6.49$ (s, 1H).

${ }^{13} \mathrm{C} \mathrm{NMR}\left(151 \mathrm{MHz}, \mathrm{CDCl}_{3}\right) \delta 145.26,144.22,142.02,141.36,139.81,138.99,138.77,138.72$, 136.07, 128.48, 127.85, 127.35, 127.27 - 124.42 (m), $120.09-119.37$ (m), 52.79.

DP:

HRMS (DART/AccuTOF) m/z: [M] ${ }^{+}$Calcd for $\mathrm{C}_{33} \mathrm{H}_{14} \mathrm{D}_{8}$ 437.2726; Found 437.2755. 


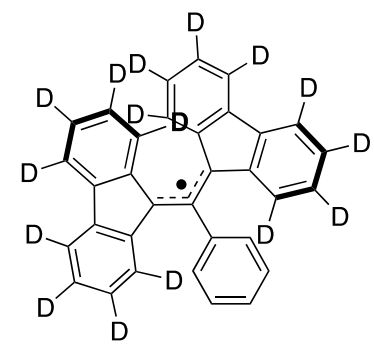

\section{$1,3-\left[\alpha, \beta, \gamma, \delta-d_{16}\right]$-BDPA radical (22)}

Prepared according to the general procedure used for compound 14. 'BuOK (47 mg, $0.42 \mathrm{mmol})$ was added in portions to a stirred solution of compound 9-( $(9 H$-fluoren-9-yl-1,2,3,4,5,6,7,8$d_{8}$ )(phenyl)methylene)-9H-fluorene-1,2,3,4,5,6,7,8- $d_{8}$ (30 mg, $0.07 \mathrm{mmol}$ ), $\mathrm{AgNO}_{3}$ (71 mg, 0.42 $\mathrm{mmol})$, DMF $(0.35 \mathrm{~mL})$ was used. Yielding 1,3-[ $\left.d_{16}\right]$-BDPA radical as a deep red glassy solid (22 $\mathrm{mg}, 72 \%)$.

\section{NMR Spectra}

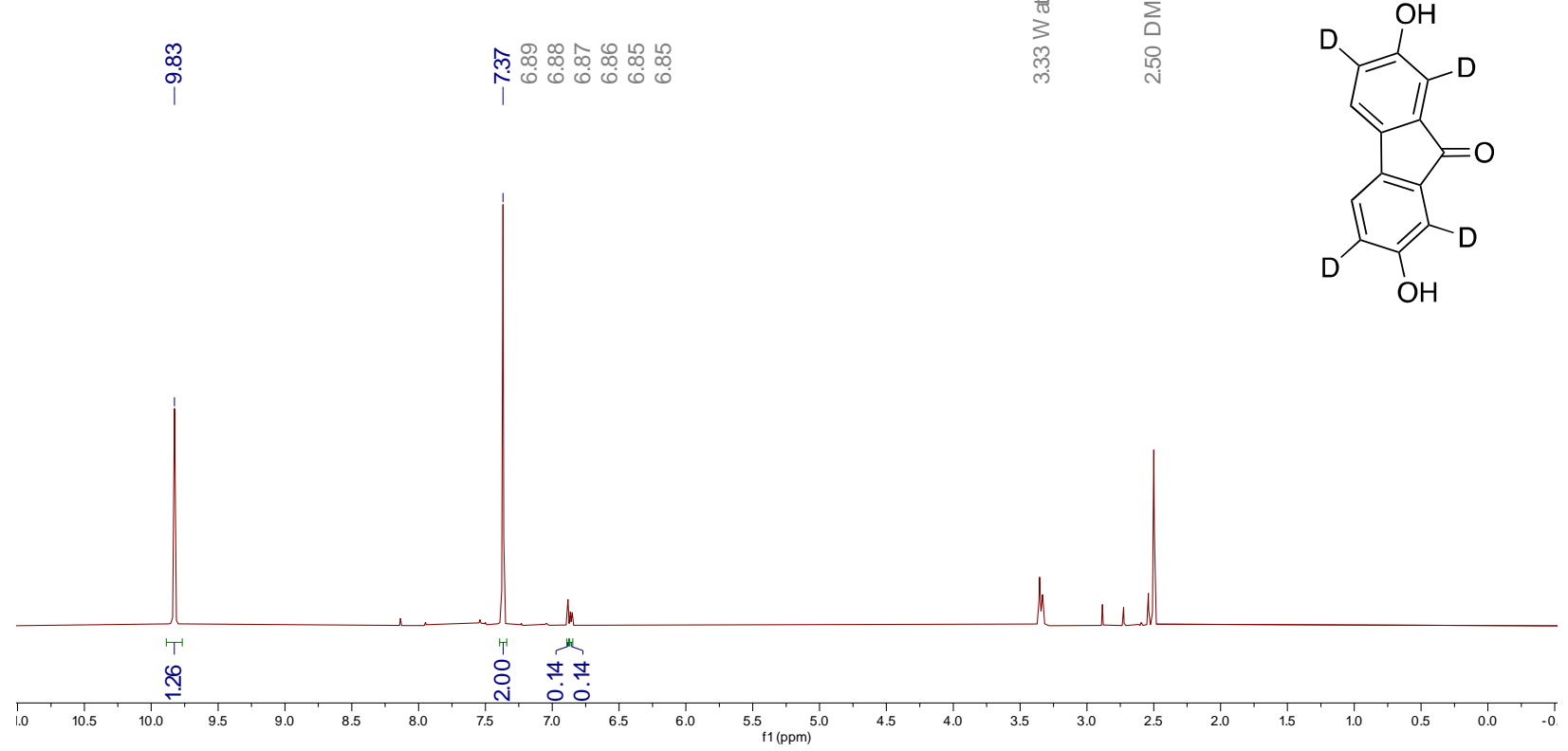

Figure $\mathbf{S 4}{ }^{1} \mathrm{H}$ NMR spectrum (600 MHz, DMSO- $\left.d_{6}\right)$ of 2,7-dihydroxy-9H-fluoren-9-one$1,3,6,8-d_{4}(2)$ 


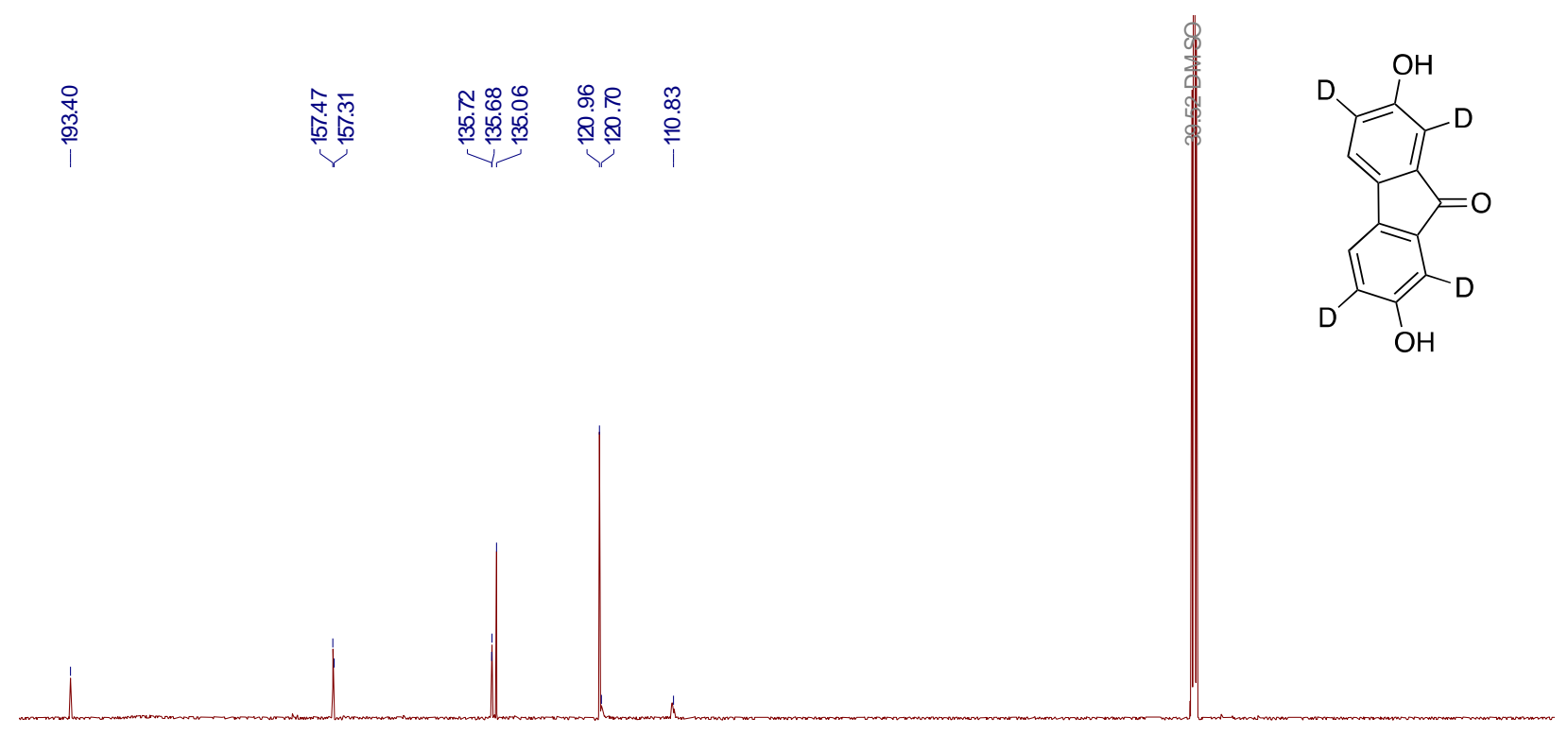

Figure S5 ${ }^{13} \mathrm{C}$ NMR spectrum (151 MHz, DMSO-d 6 ) of 2,7-dihydroxy-9H-fluoren-9-one$1,3,6,8-d_{4}(2)$<smiles>[2H]c1cc2c(c([2H])c1[2H])C(=O)c1c-2cc([2H])c(OCC)c1[2H]</smiles>
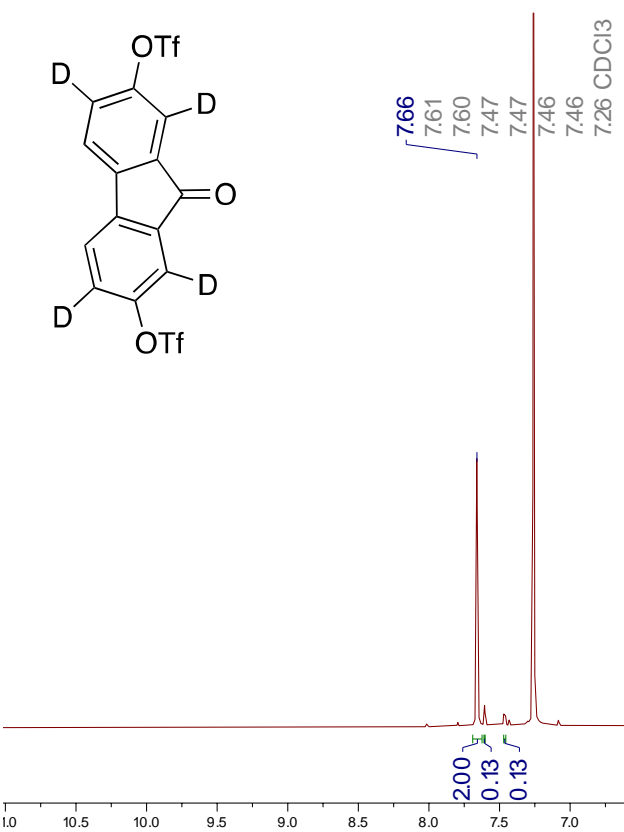

Figure S6 ${ }^{1} \mathrm{H}$ NMR spectrum $\left(600 \mathrm{MHz}, \mathrm{CDCl}_{3}\right)$ of 9-oxo-9H-fluorene-2,7-diyl-1,3,6,8- $\boldsymbol{d}_{\mathbf{4}}$ bis(trifluoromethanesulfonate) (3) 


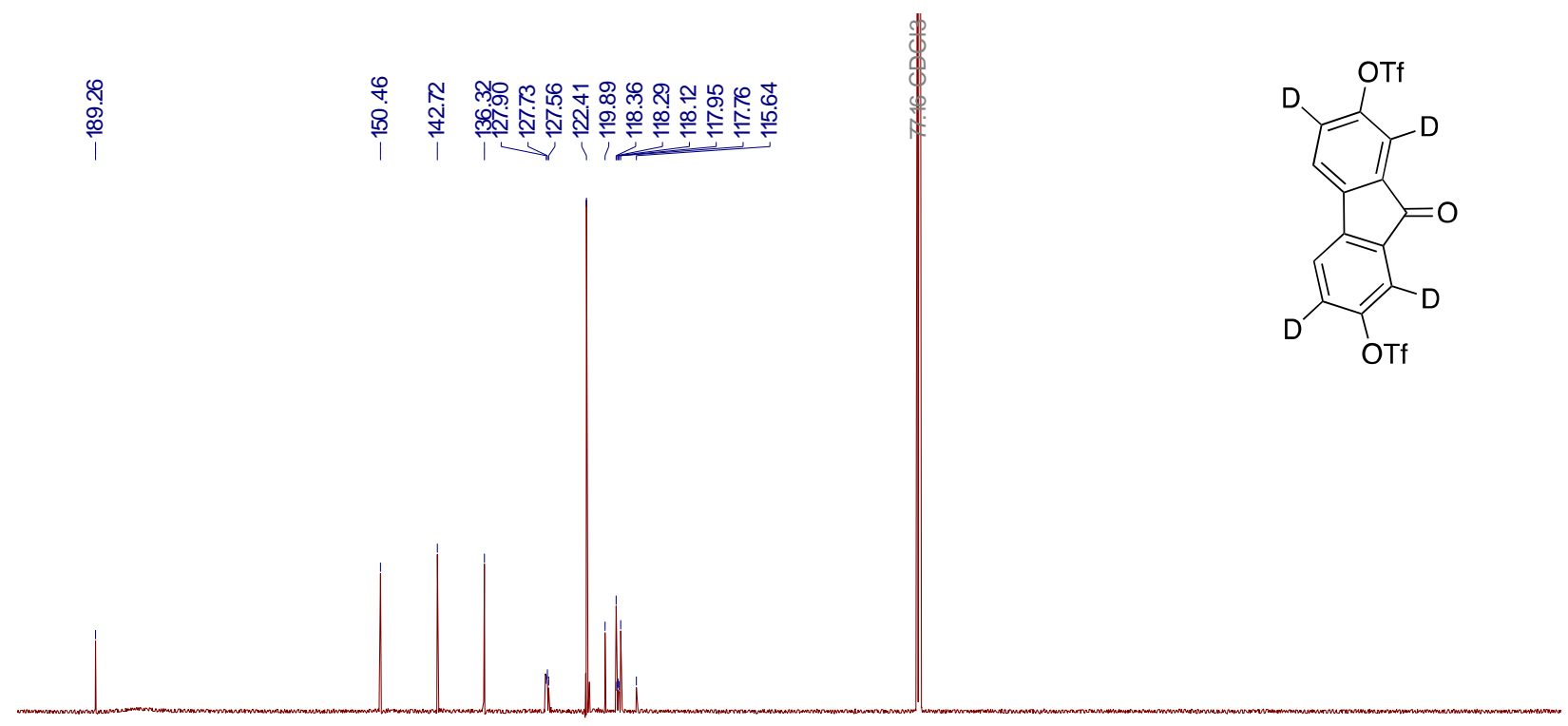

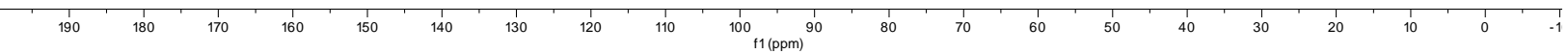

Figure S7 ${ }^{13} \mathrm{C}$ NMR spectrum (151 $\left.\mathrm{MHz}, \mathrm{CDCl}_{3}\right)$ of 9-oxo-9H-fluorene-2,7-diyl-1,3,6,8- $\boldsymbol{d}_{\mathbf{4}}$ bis(trifluoromethanesulfonate) (3)

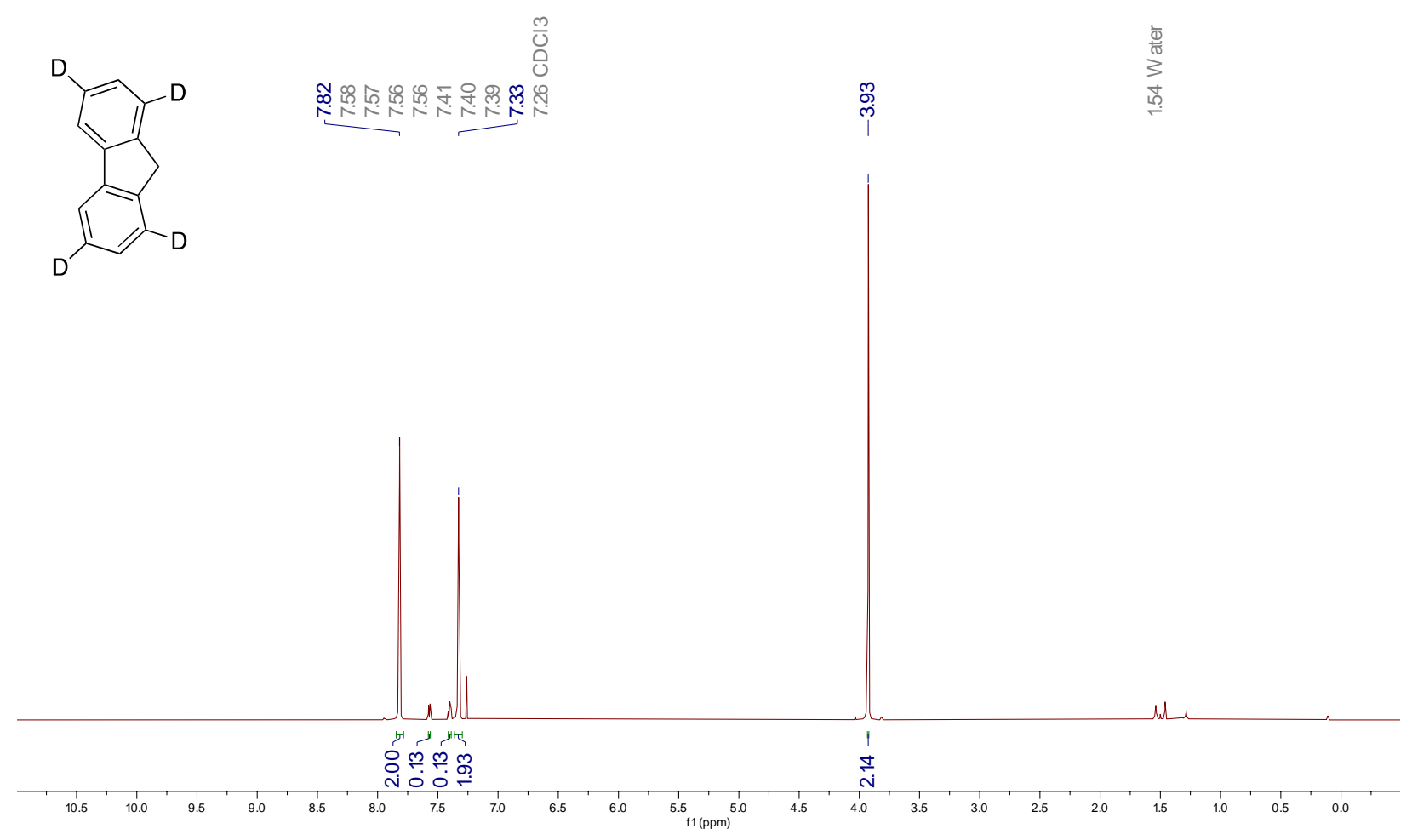

Figure S8 ${ }^{1} \mathrm{H}$ NMR spectrum $\left(600 \mathrm{MHz}, \mathrm{CDCl}_{3}\right)$ of $\mathbf{9 H}$-fluorene-1,3,6,8-d $\mathbf{( 4 )}$ 


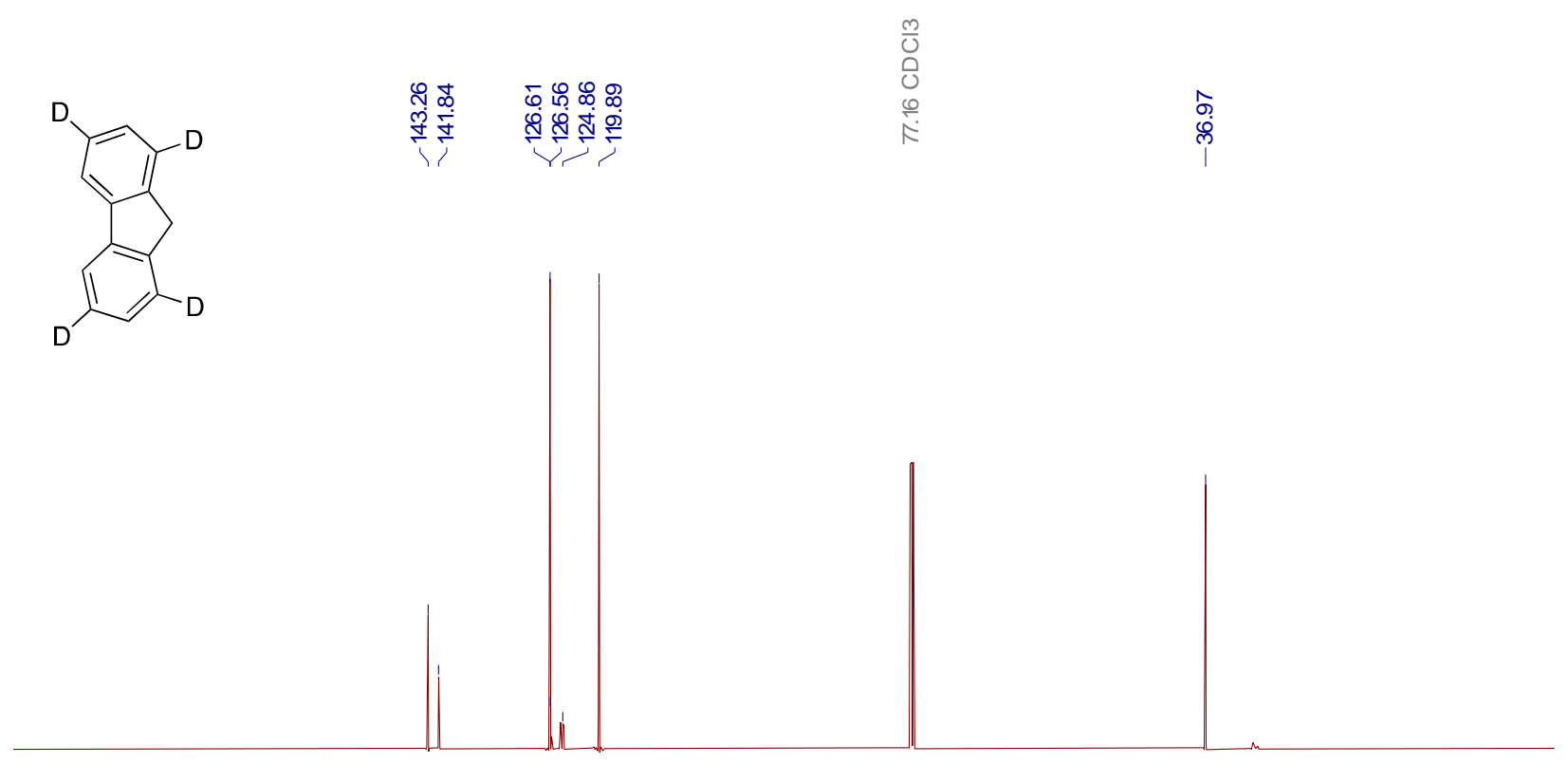

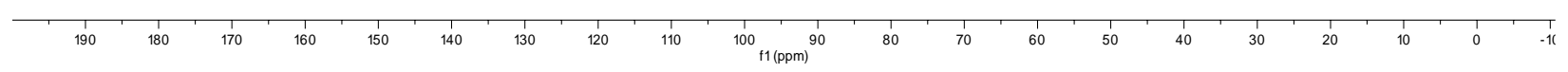

Figure S9 ${ }^{13} \mathrm{C}$ NMR spectrum (151 MHz, $\left.\mathrm{CDCl}_{3}\right)$ of $\mathbf{9 H}$-fluorene-1,3,6,8- $\boldsymbol{d}_{\mathbf{4}}$ (4)

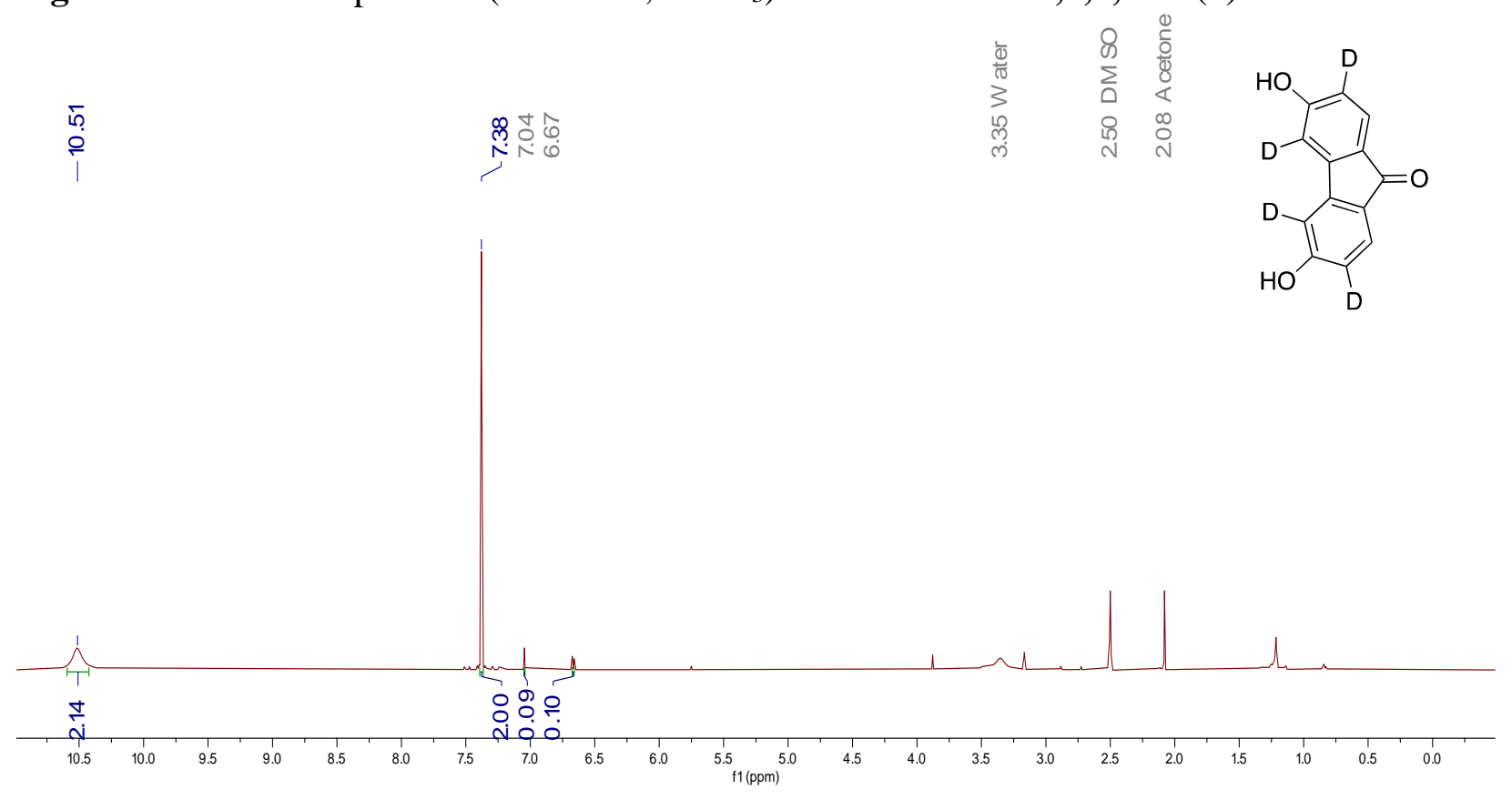

Figure S10 ${ }^{1} \mathrm{H}$ NMR spectrum (600 MHz, DMSO- $\left.d_{6}\right)$ of 3,6-dihydroxy-9H-fluoren-9-one$2,4,5,7-d_{4}(8)$ 

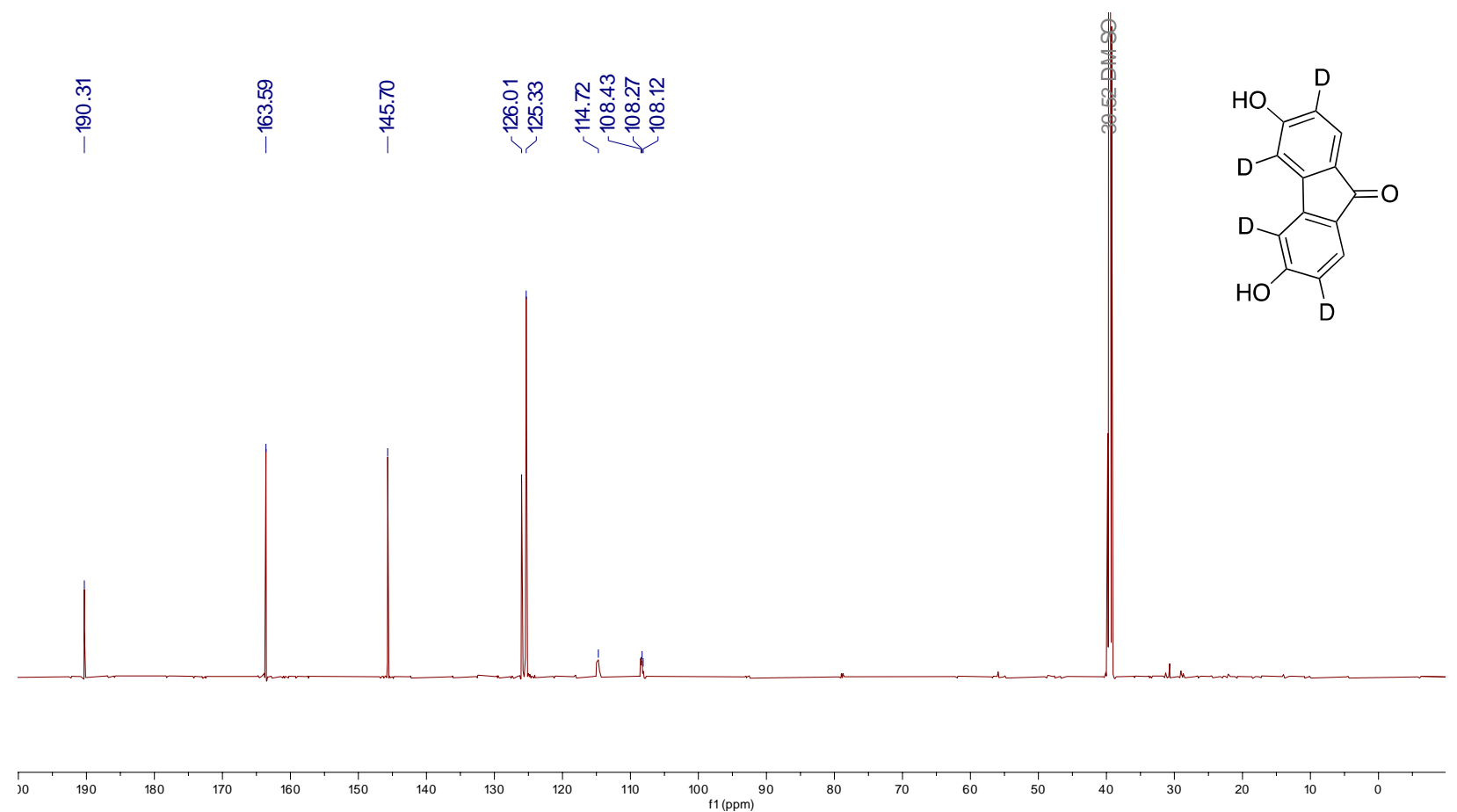

Figure S11 ${ }^{13} \mathrm{C}$ NMR spectrum (151 MHz, DMSO- $\left.d_{6}\right)$ of 3,6-dihydroxy-9H-fluoren-9-one2,4,5,7-d4 (8)

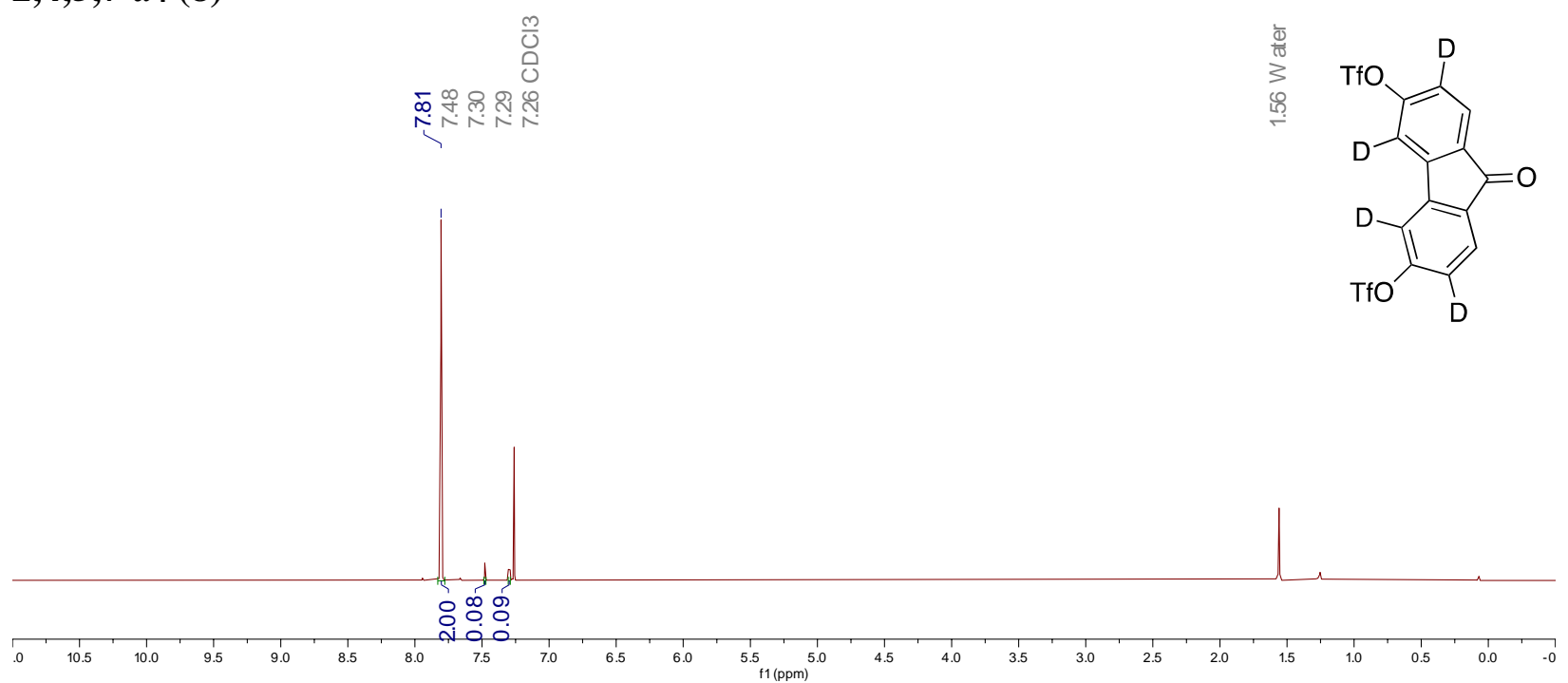

Figure $\mathbf{S 1 2}{ }^{1} \mathrm{H}$ NMR spectrum $\left(600 \mathrm{MHz}, \mathrm{CDCl}_{3}\right)$ of 9-oxo-9H-fluorene-3,6-diyl-2,4,5,7-d bis(trifluoromethanesulfonate) (9) 

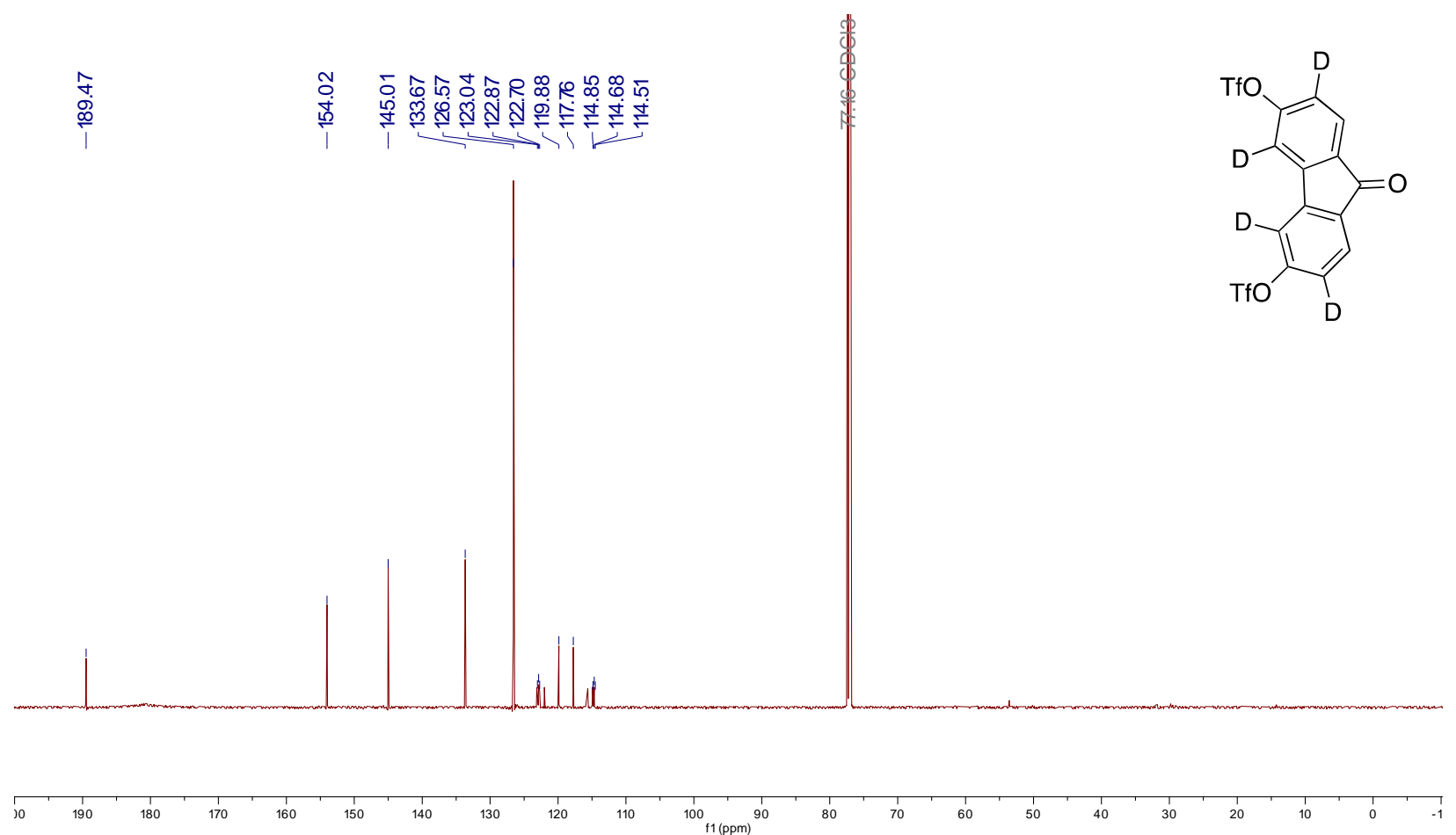

Figure S13 ${ }^{13} \mathrm{C}$ NMR spectrum $\left(151 \mathrm{MHz}, \mathrm{CDCl}_{3}\right)$ of 9-oxo-9H-fluorene-3,6-diyl-2,4,5,7-d bis(trifluoromethanesulfonate) (9)

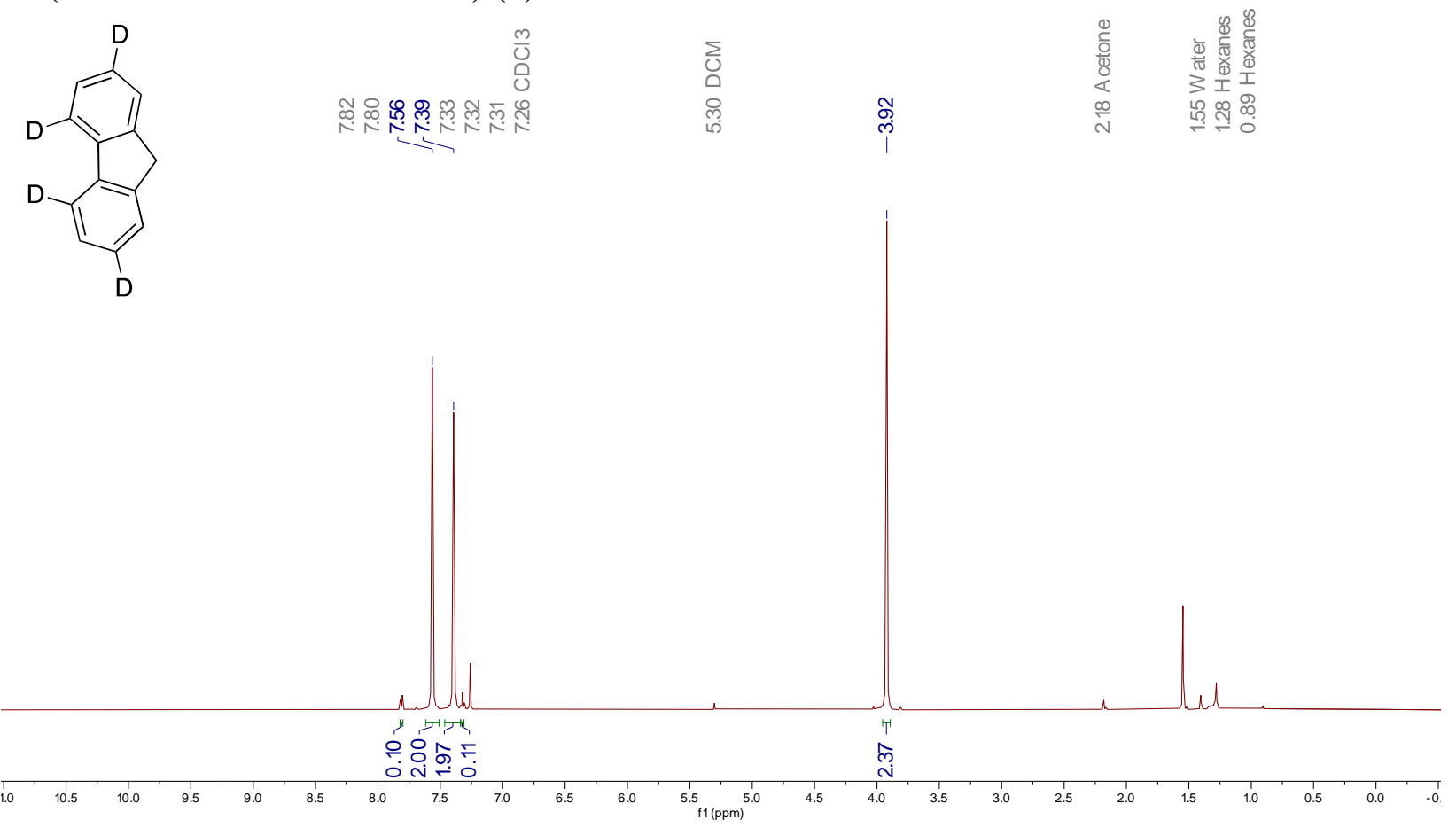

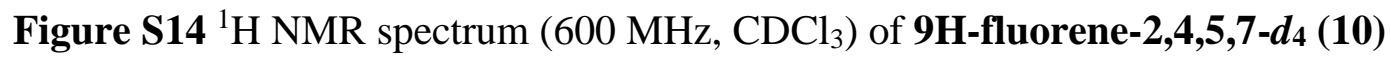




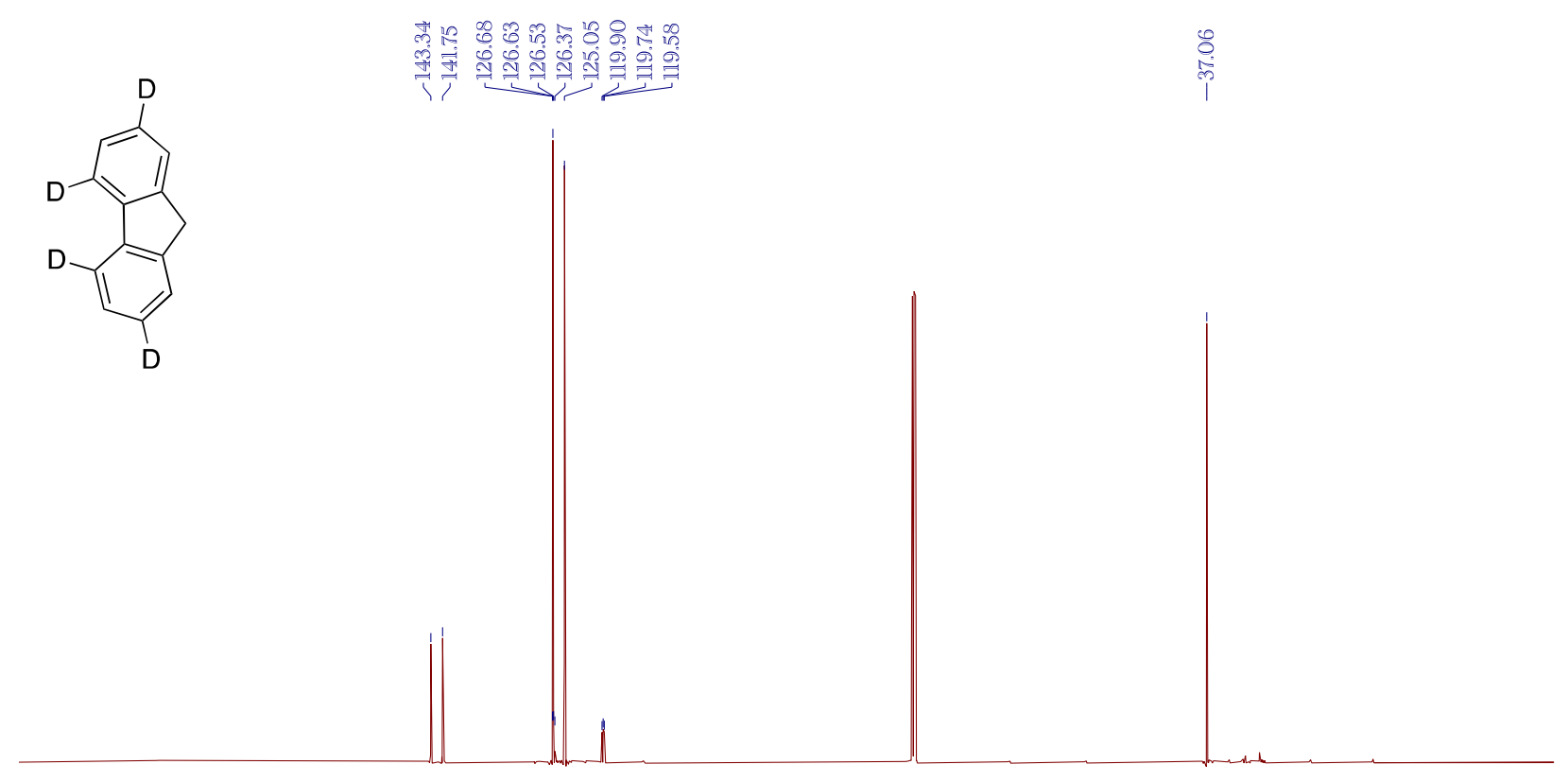

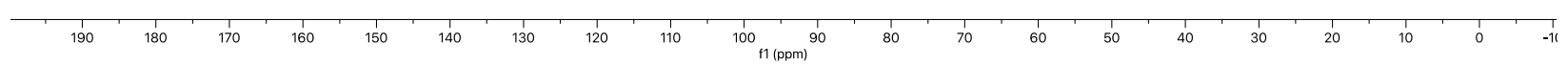

Figure S15 ${ }^{13} \mathrm{C}$ NMR spectrum (151 $\left.\mathrm{MHz}, \mathrm{CDCl}_{3}\right)$ of 9H-fluorene-2,4,5,7-d4 (10)

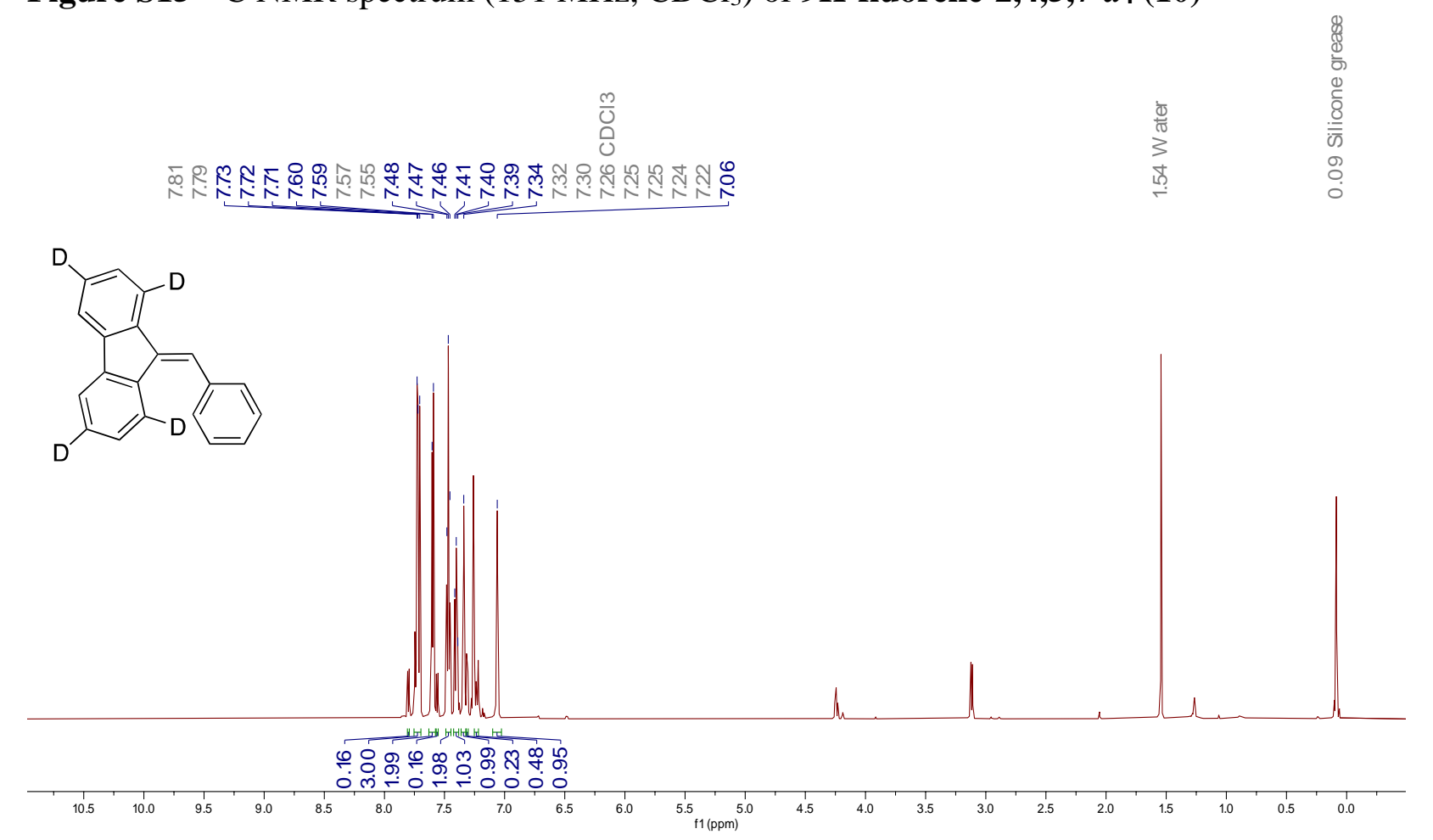

Figure S16 ${ }^{1} \mathrm{H}$ NMR spectrum $\left(600 \mathrm{MHz}, \mathrm{CDCl}_{3}\right)$ of 9-benzylidene-9H-fluorene-1,3,6,8- $\boldsymbol{d} \mathbf{4}(\mathbf{1 1})$ 

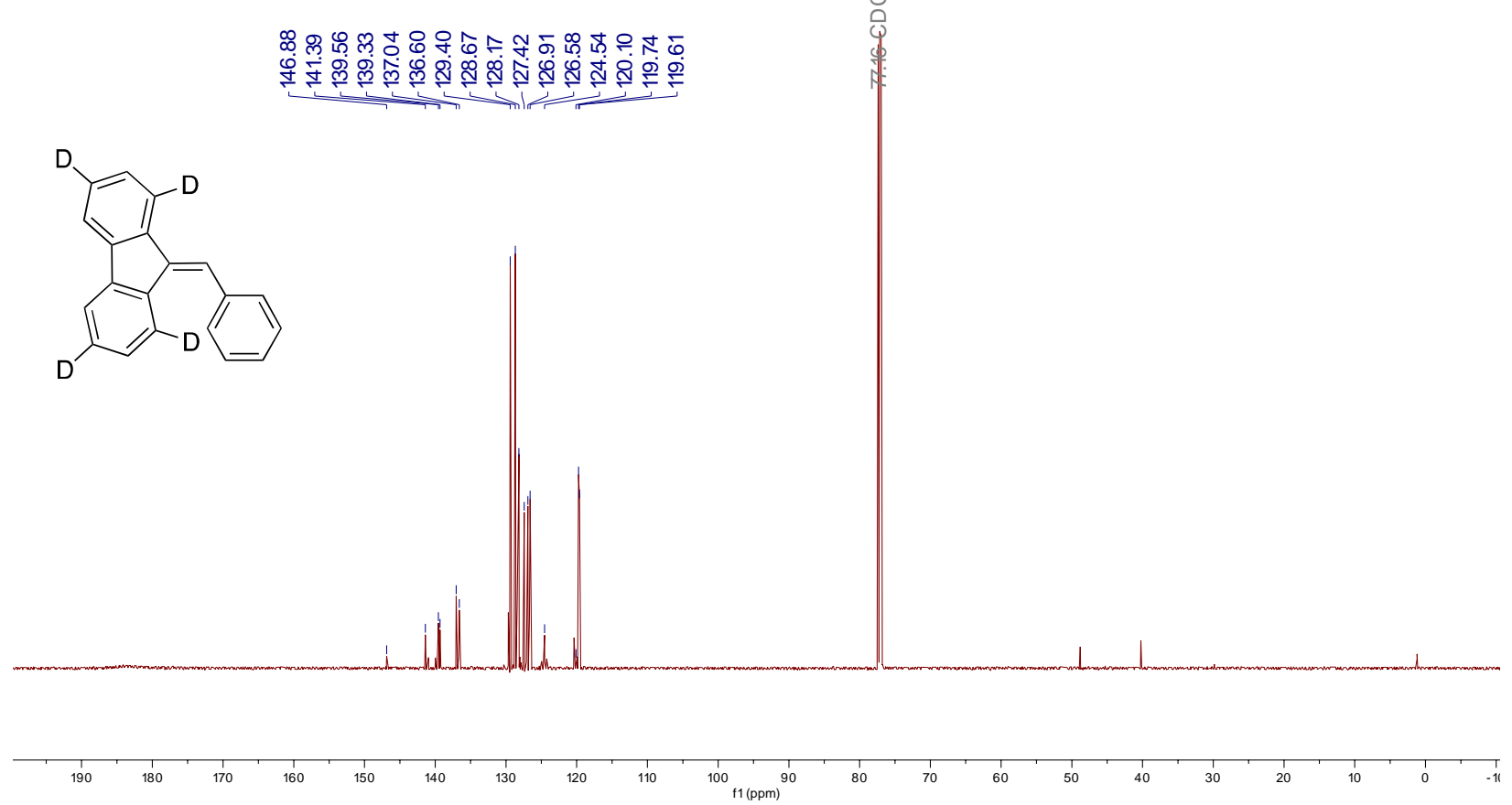

Figure S17 ${ }^{13} \mathrm{C}$ NMR spectrum (151 MHz, $\mathrm{CDCl}_{3}$ ) of 9-benzylidene-9H-fluorene-1,3,6,8- $\boldsymbol{d}_{\mathbf{4}}(\mathbf{1 1})$
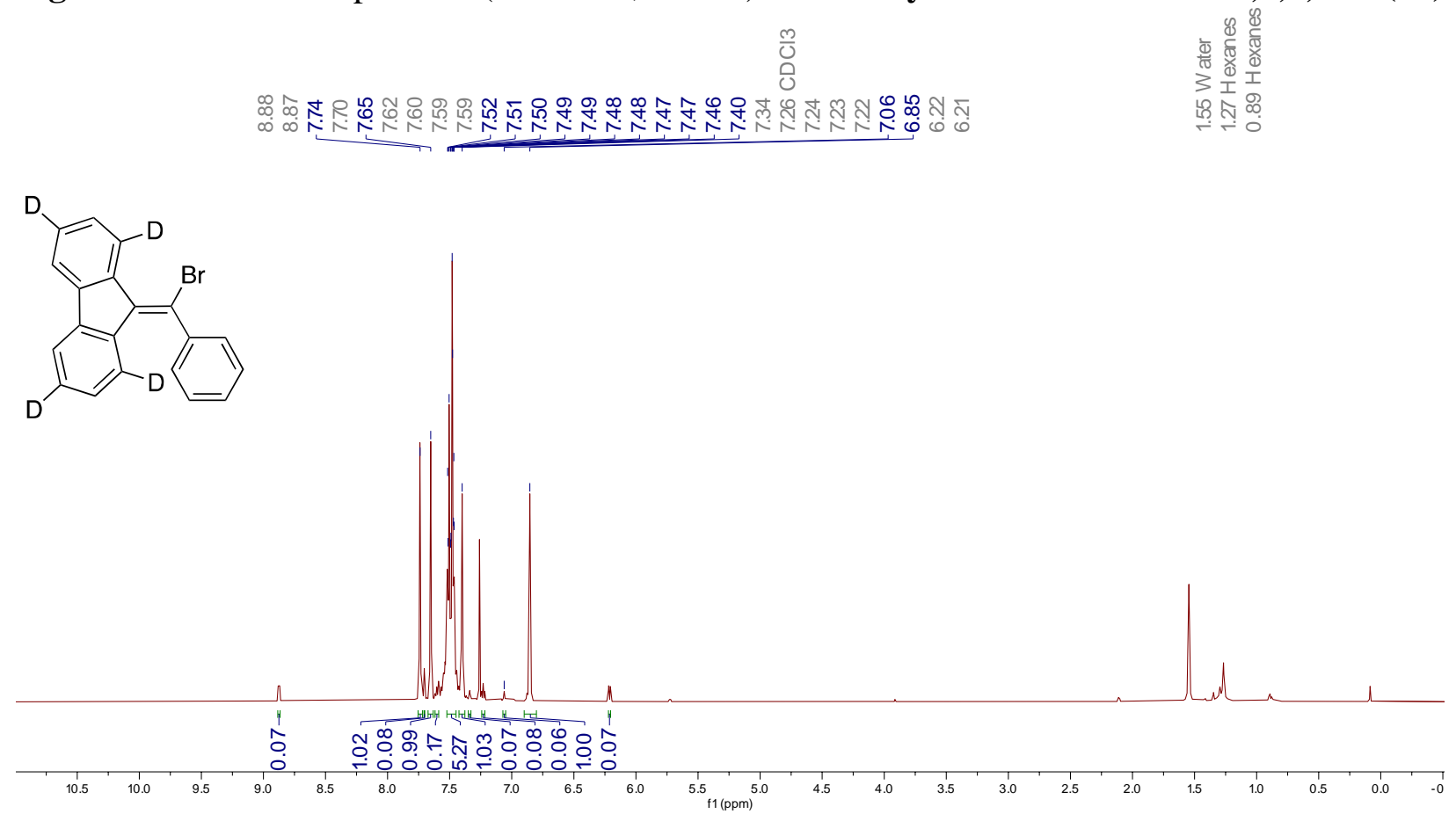

Figure S18 ${ }^{1} \mathrm{H}$ NMR spectrum $\left(600 \mathrm{MHz}, \mathrm{CDCl}_{3}\right)$ of 9-(bromo(phenyl)methylene)-9Hfluorene-1,3,6,8-d4 (12) 


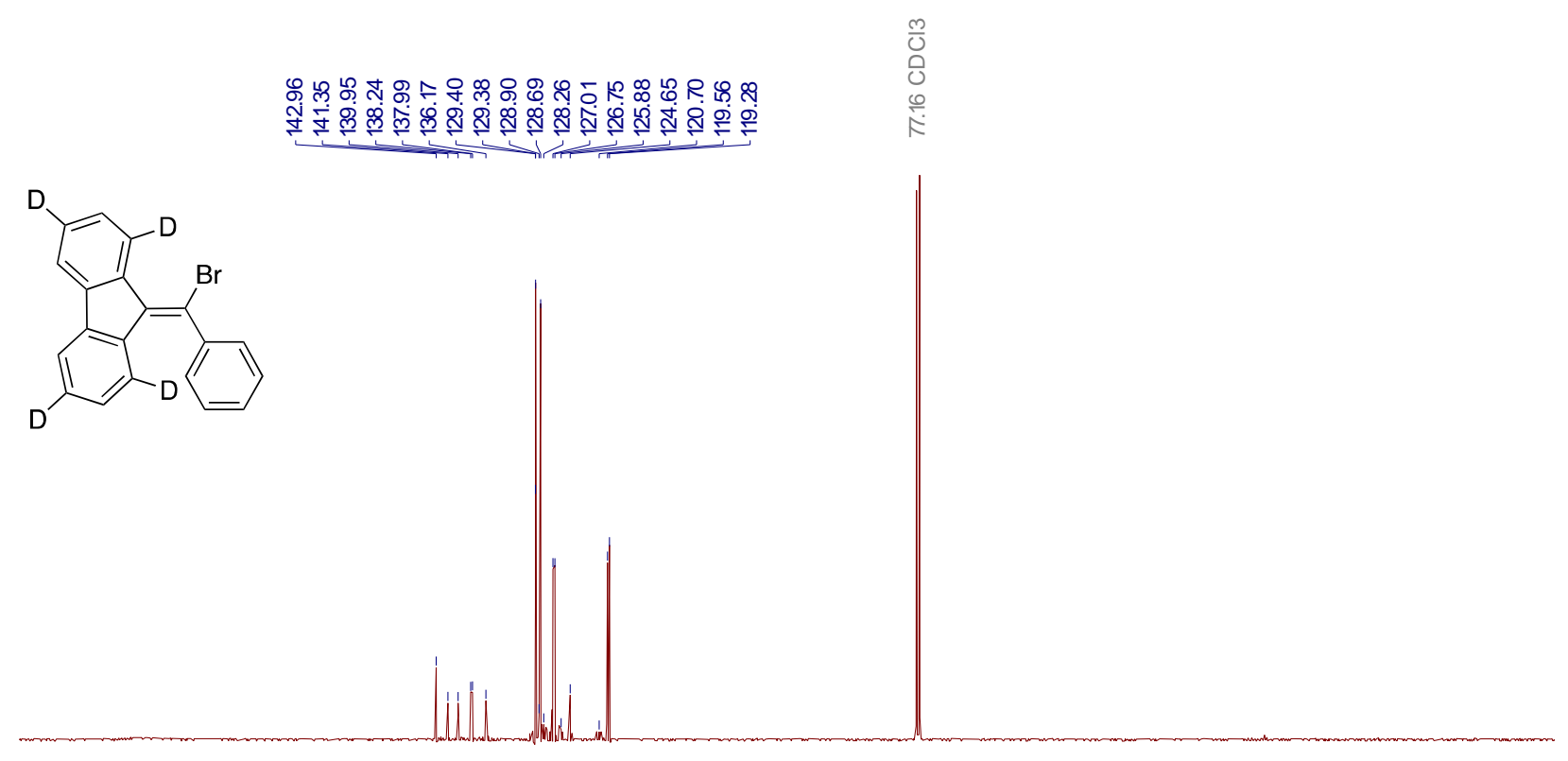

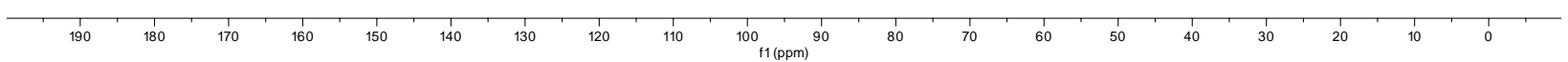

Figure $\mathbf{S 1 9}{ }^{13} \mathrm{C}$ NMR spectrum $\left(151 \mathrm{MHz}, \mathrm{CDCl}_{3}\right)$ of 9-(bromo(phenyl)methylene)-9Hfluorene-1,3,6,8- $d_{4}(12)$
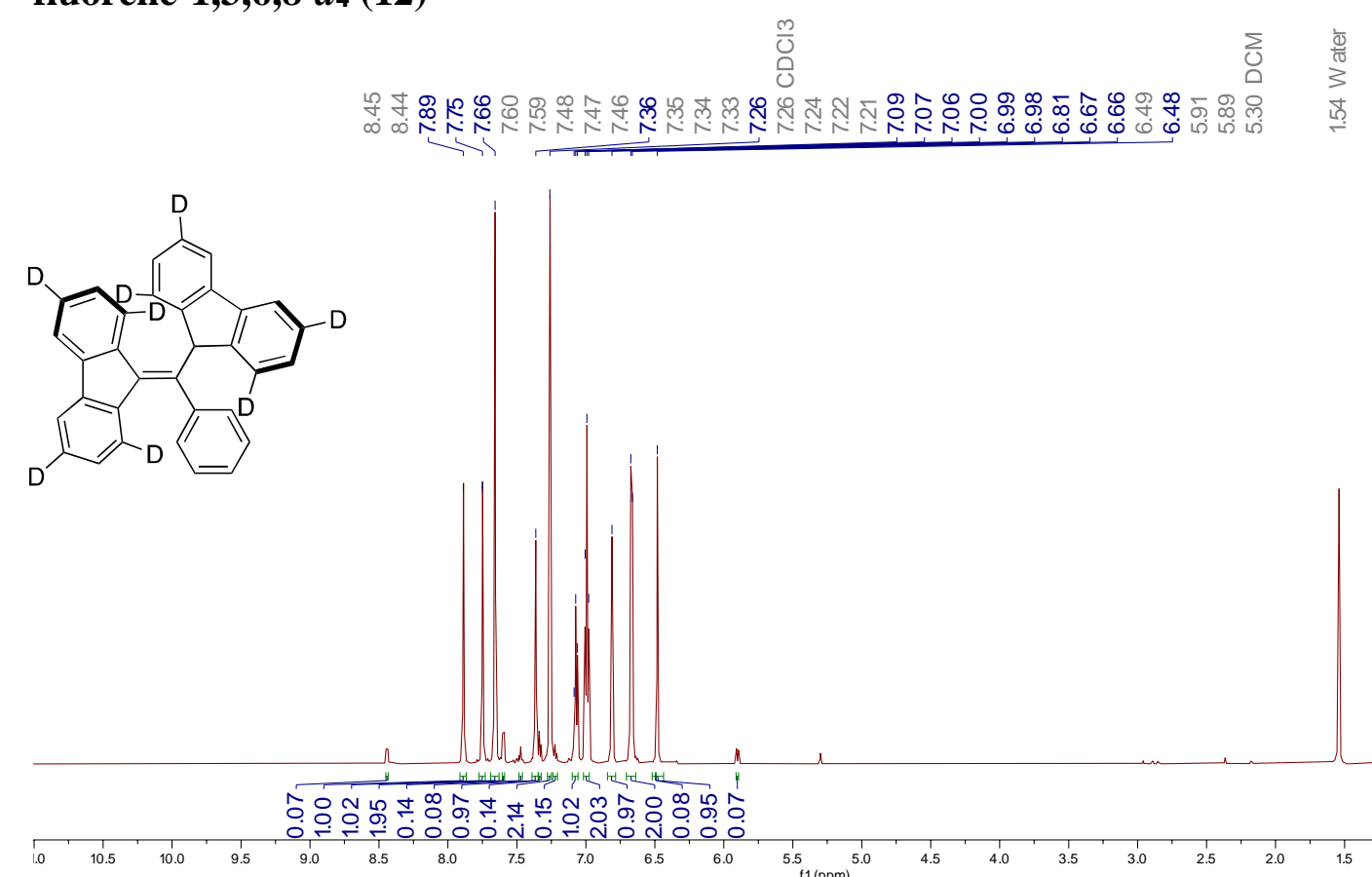

Figure S20 ${ }^{1} \mathrm{H} \quad \mathrm{NMR}$ spectrum $\left(600 \quad \mathrm{MHz}, \mathrm{CDCl}_{3}\right)$ of 9-((9H-fluoren-9-yl-1,3,6,8$\left.d_{4}\right)\left(\right.$ phenyl)methylene)-9H-fluorene-1,3,6,8- $d_{4}(13)$ 

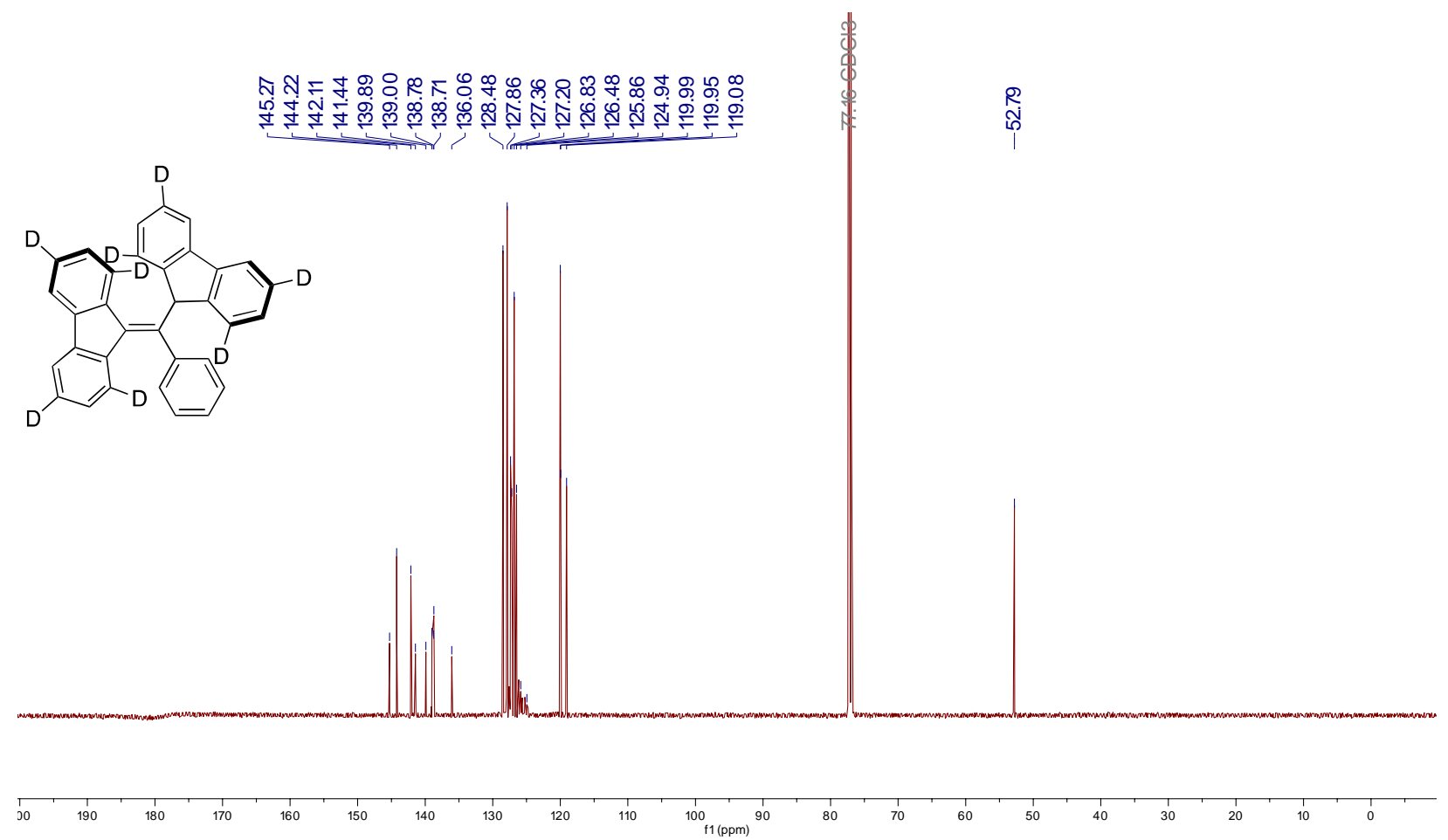

Figure S21 ${ }^{13} \mathrm{C}$ NMR spectrum $\left(151 \quad \mathrm{MHz}, \mathrm{CDCl}_{3}\right)$ of 9-((9H-fluoren-9-yl-1,3,6,8$\left.d_{4}\right)$ (phenyl)methylene)-9H-fluorene-1,3,6,8- $d_{4}(13)$
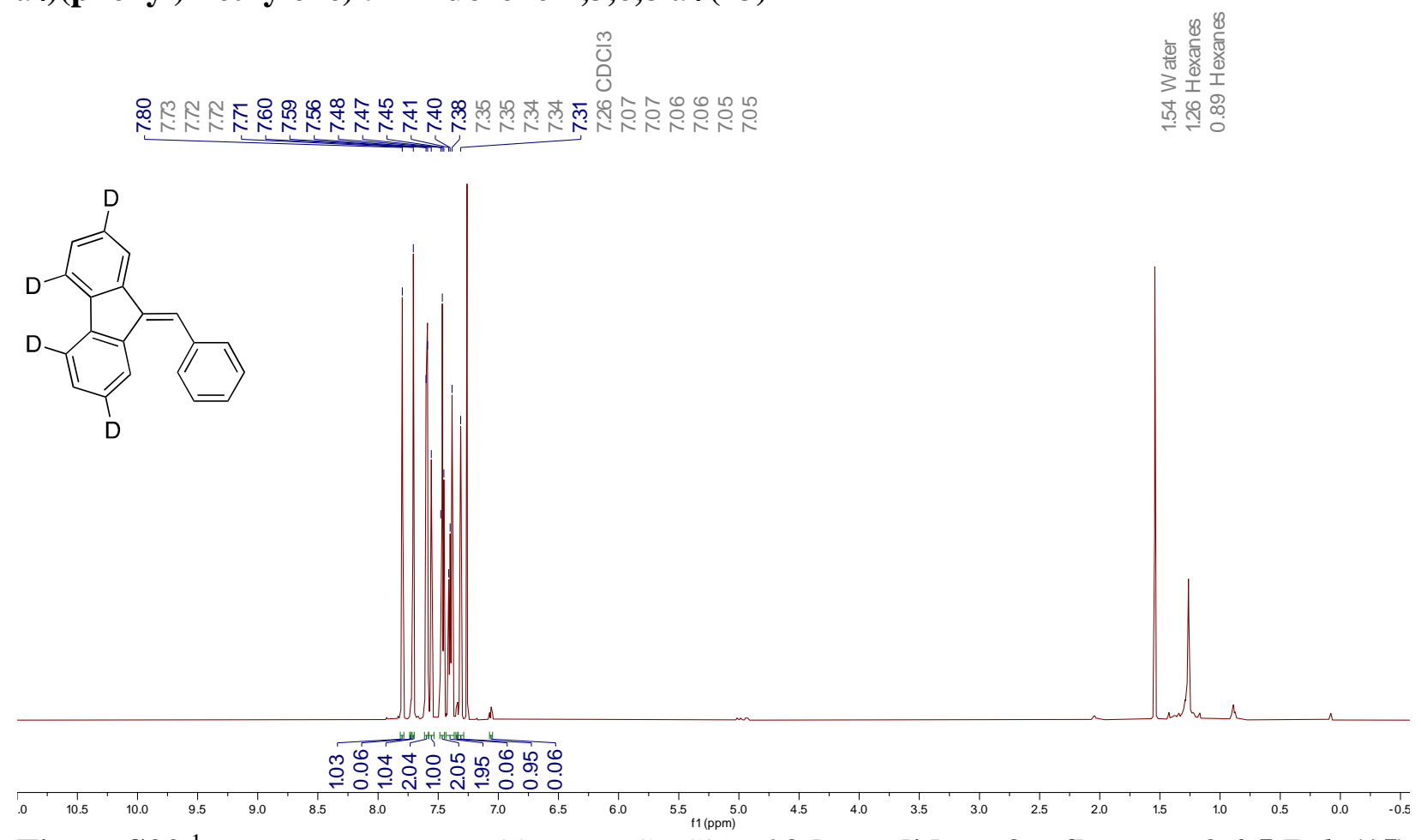

Figure S22 ${ }^{1} \mathrm{H}$ NMR spectrum $\left(600 \mathrm{MHz}, \mathrm{CDCl}_{3}\right)$ of 9-benzylidene-9H-fluorene-2,4,5,7-d $\mathbf{4}(\mathbf{1 5})$ 


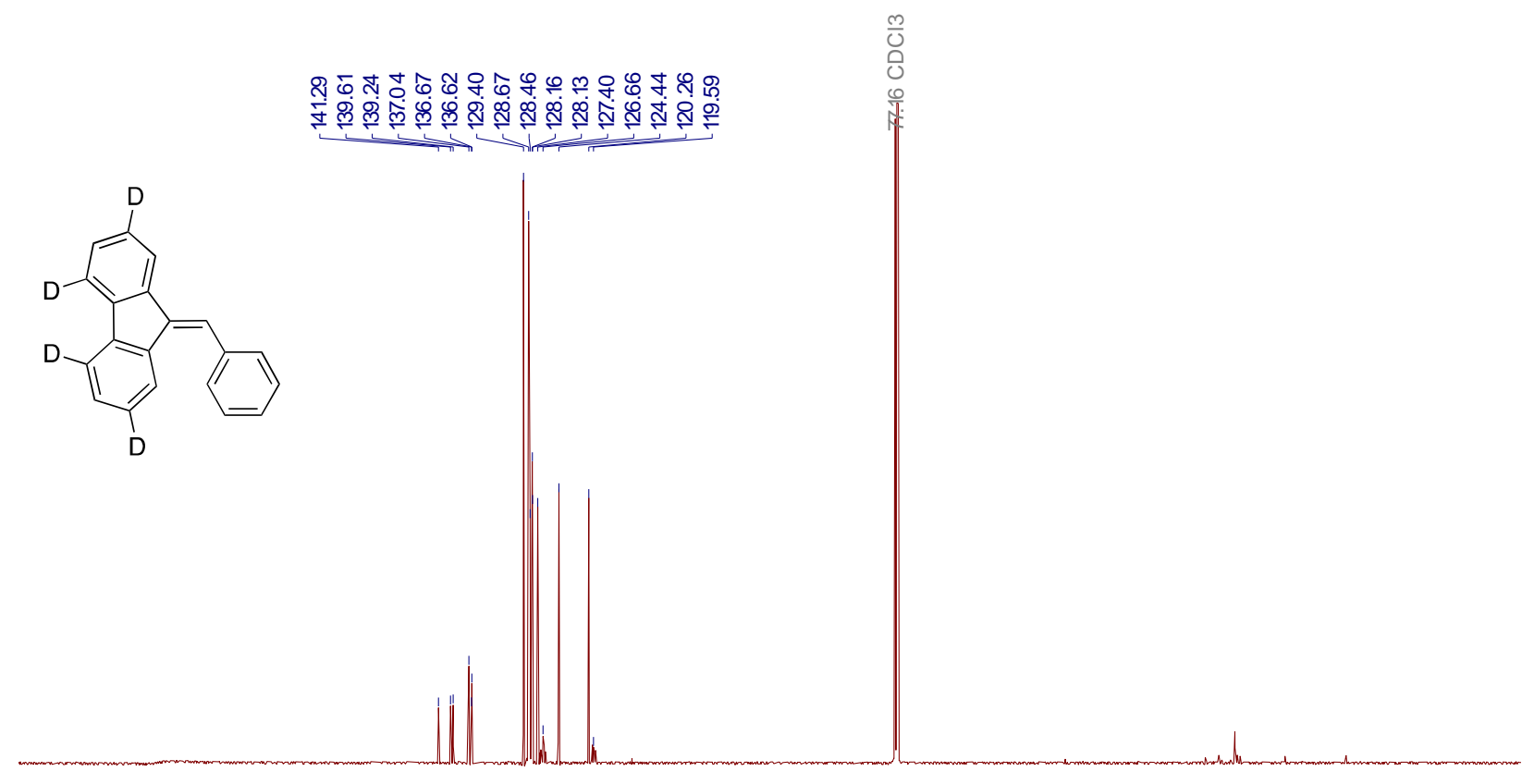

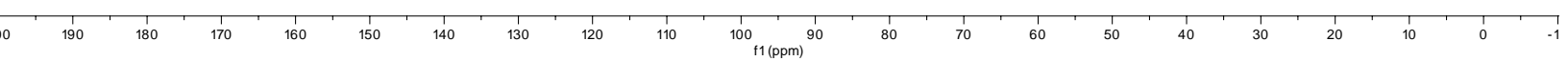

Figure $\mathbf{S 2 3}{ }^{13} \mathrm{C}$ NMR spectrum (151 MHz, $\mathrm{CDCl}_{3}$ ) of 9-benzylidene-9H-fluorene-2,4,5,7-d4 (15)
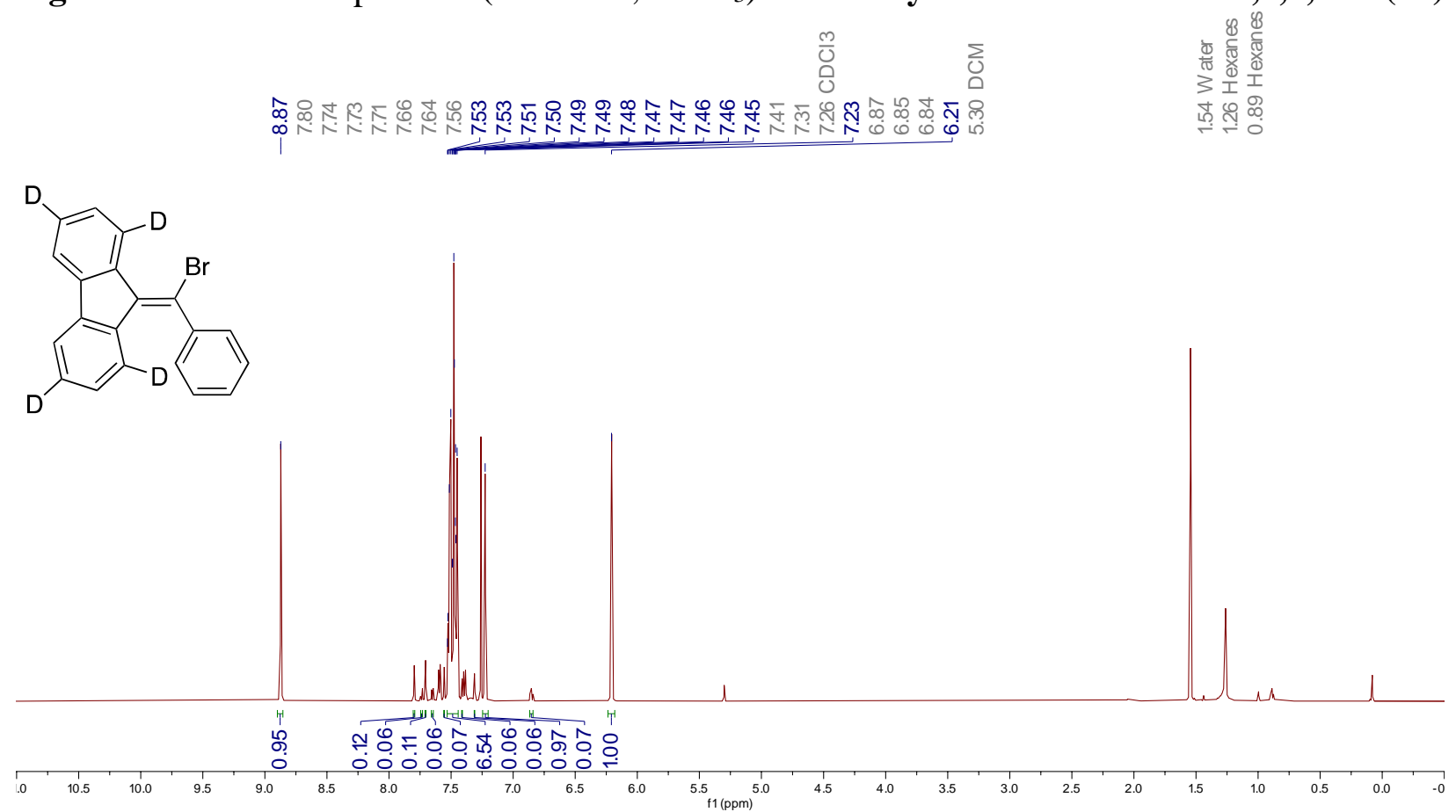

Figure S24 ${ }^{1} \mathrm{H}$ NMR spectrum $\left(600 \mathrm{MHz}, \mathrm{CDCl}_{3}\right)$ of 9-(bromo(phenyl)methylene)-9Hfluorene-2,4,5,7-d4 (16) 


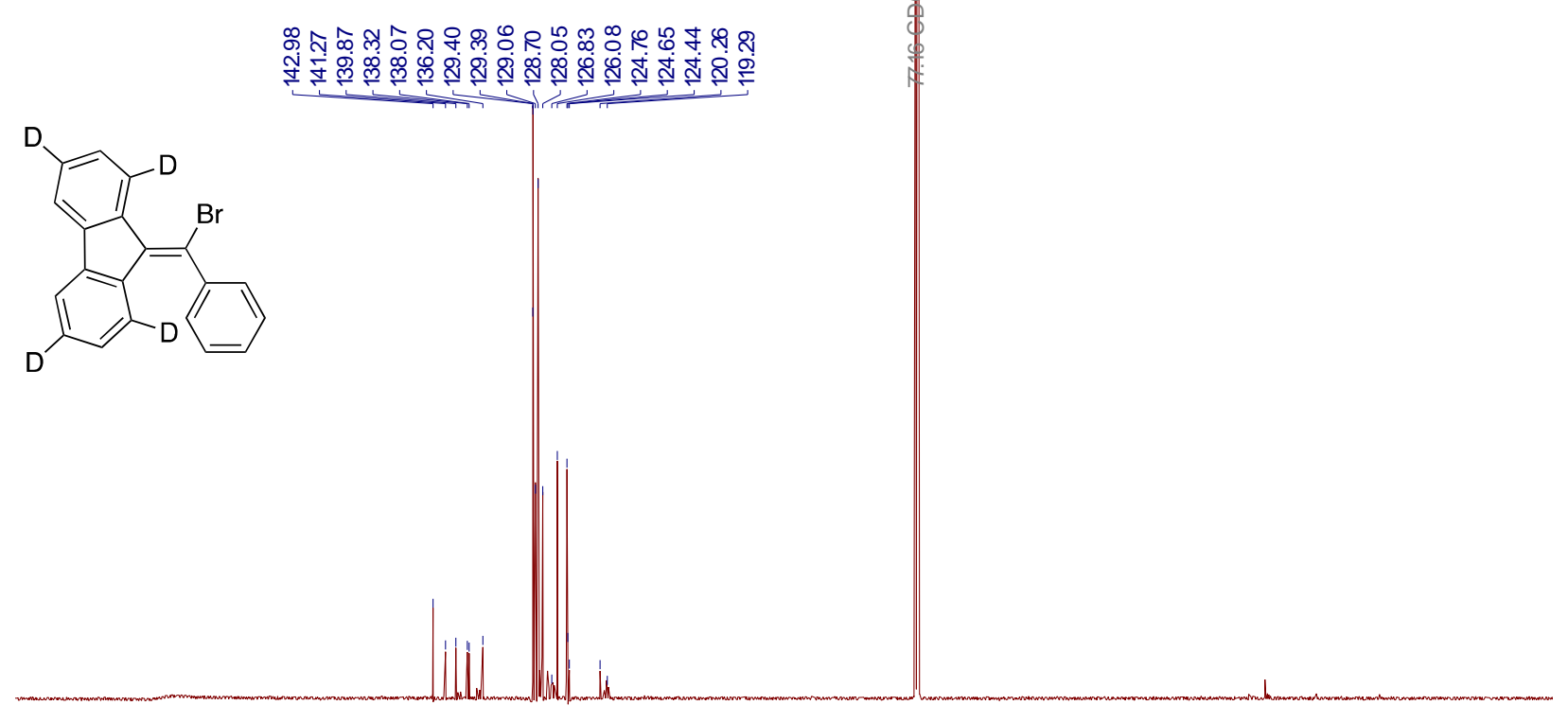

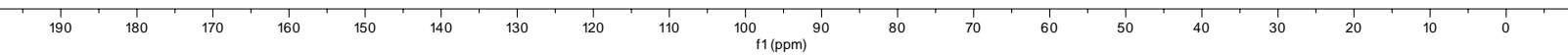

Figure $\mathbf{S 2 5}{ }^{13} \mathrm{C}$ NMR spectrum (151 $\left.\mathrm{MHz} \mathrm{CDCl}_{3}\right)$ of 9-(bromo(phenyl)methylene)-9Hfluorene-2,4,5,7-d4 (16)

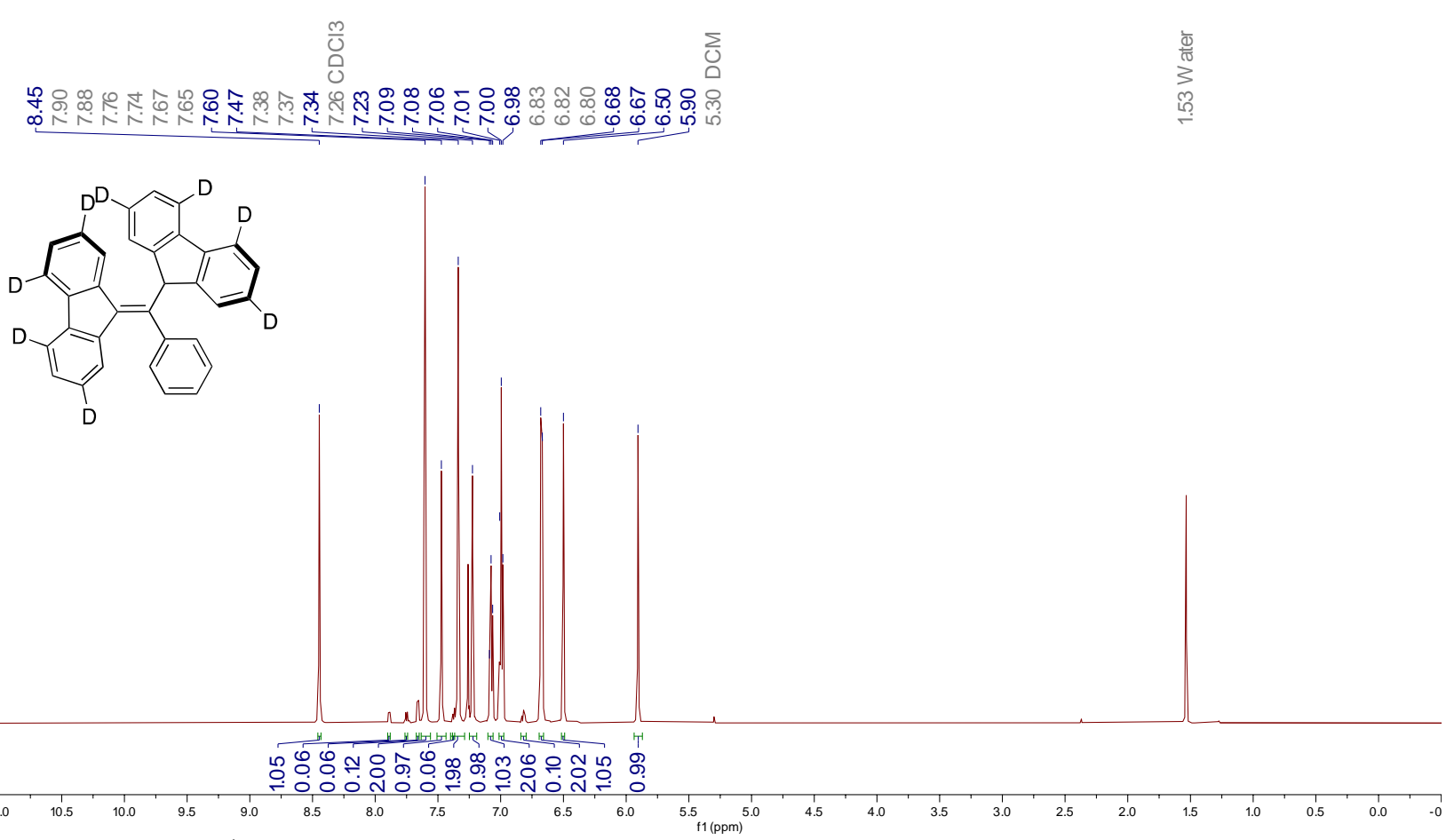

Figure $\mathbf{S 2 6}{ }^{1} \mathrm{H} \quad \mathrm{NMR}$ spectrum $\left(600 \mathrm{MHz}, \mathrm{CDCl}_{3}\right)$ of 9-((9H-fluoren-9-yl-2,4,5,7$\left.d_{4}\right)($ phenyl)methylene)-9H-fluorene-2,4,5,7-d $(17)$ 


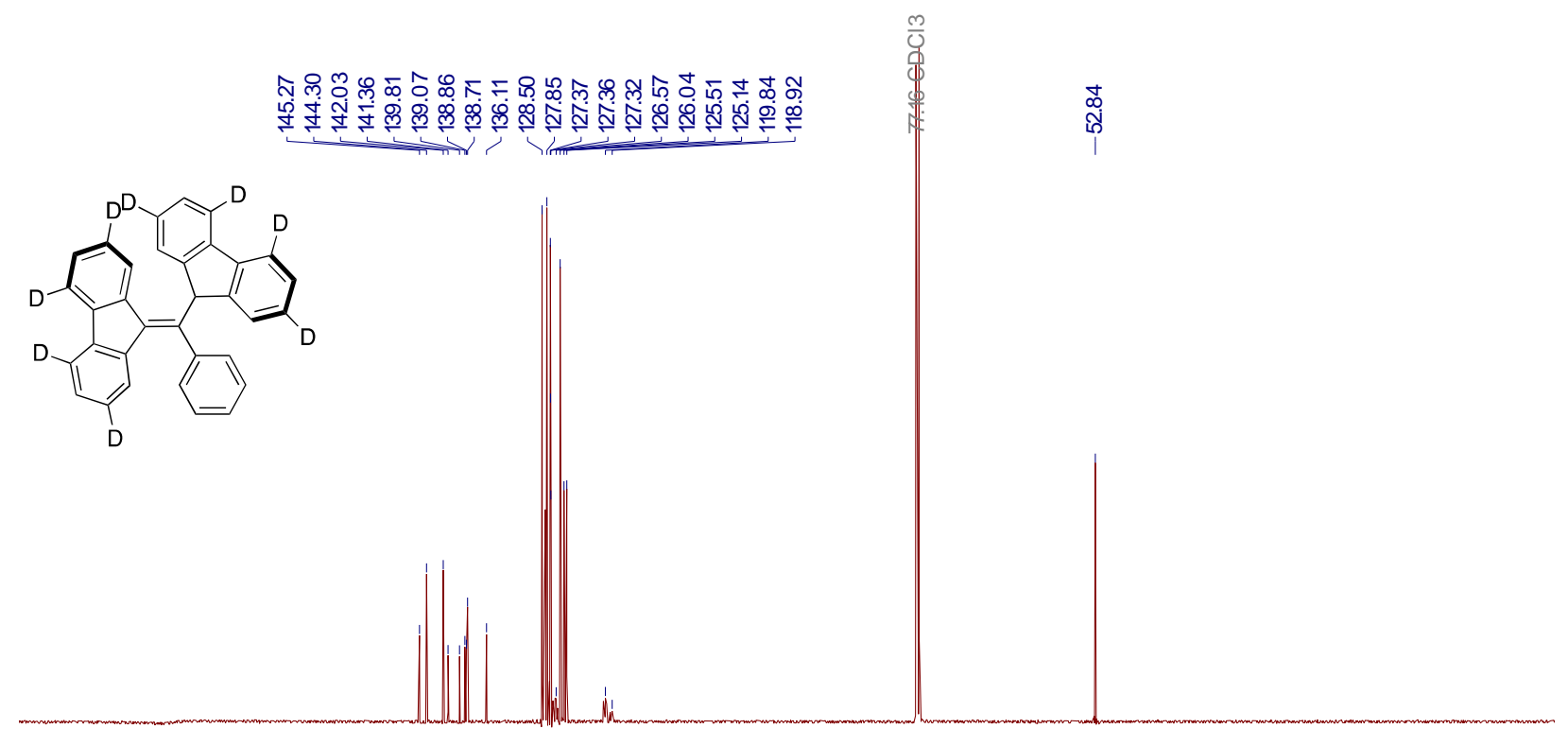

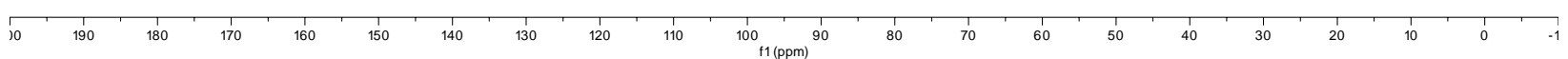

Figure S27 ${ }^{13} \mathrm{C} \quad \mathrm{NMR}$ spectrum $\left(151 \quad \mathrm{MHz}, \mathrm{CDCl}_{3}\right)$ of 9-((9H-fluoren-9-yl-2,4,5,7$\left.d_{4}\right)($ phenyl)methylene)-9H-fluorene-2,4,5,7-d4 (17)

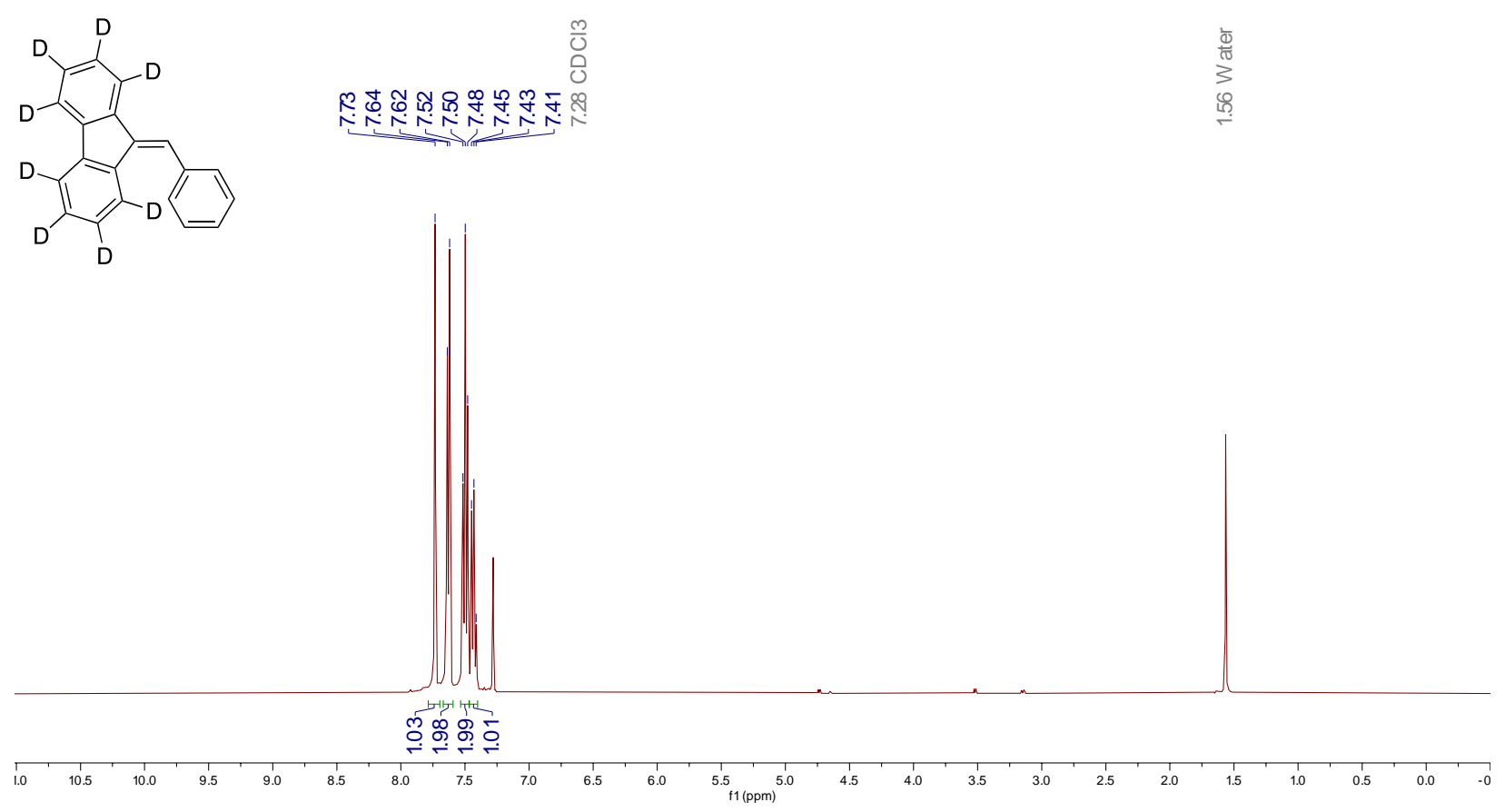

Figure S28 ${ }^{1} \mathrm{H}$ NMR spectrum $\left(600 \mathrm{MHz}, \mathrm{CDCl}_{3}\right)$ of 9-benzylidene-9H-fluorene-1,2,3,4,5,6,7,8d8 (19) 

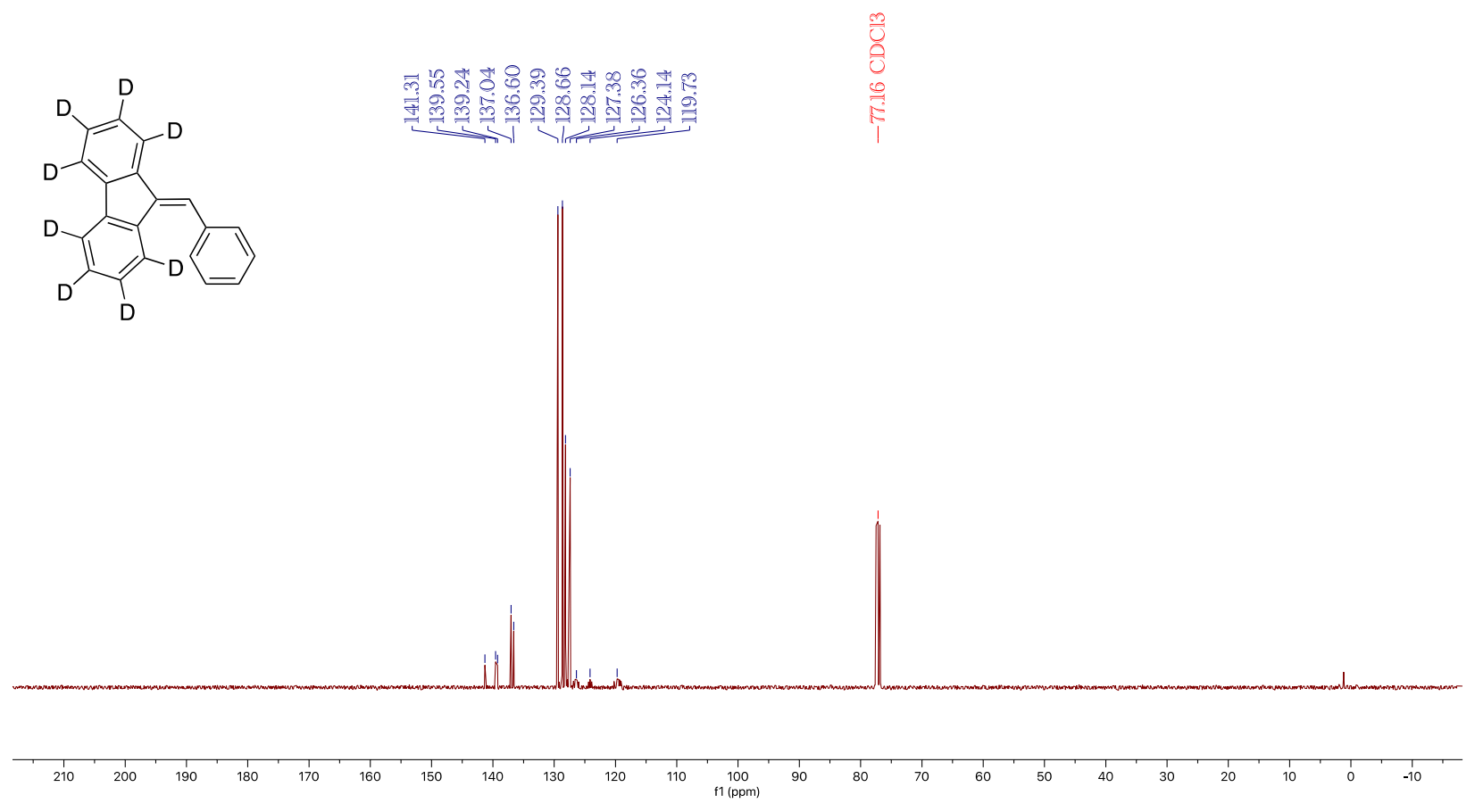

Figure S29 ${ }^{13} \mathrm{C}$ NMR spectrum (151 $\left.\mathrm{MHz}, \mathrm{CDCl}_{3}\right)$ of 9-benzylidene-9H-fluorene$1,2,3,4,5,6,7,8-d 8(19)$

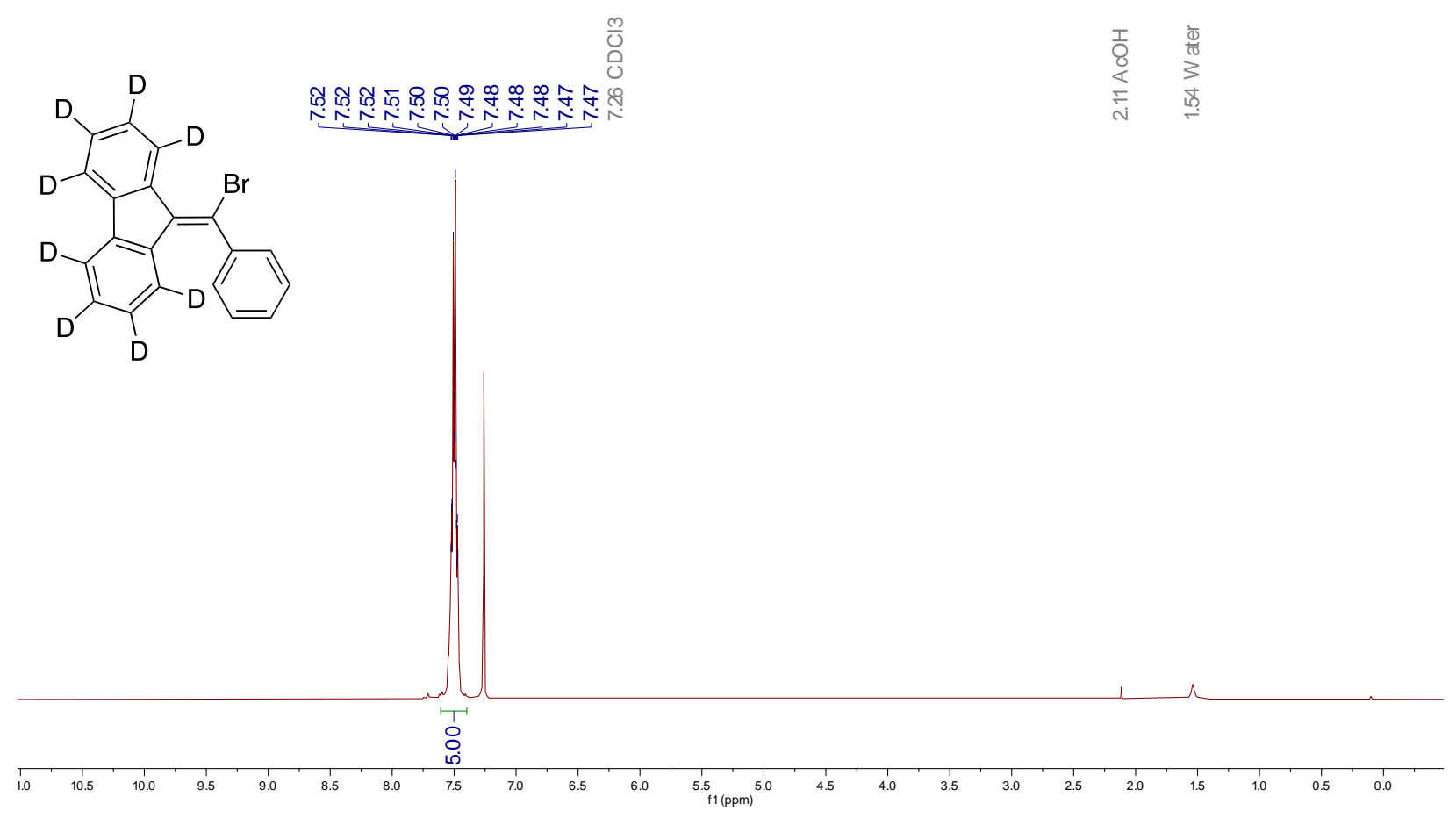


Figure S30 ${ }^{1} \mathrm{H}$ NMR spectrum $\left(600 \mathrm{MHz}, \mathrm{CDCl}_{3}\right)$ of 9-(bromo(phenyl)methylene)-9Hfluorene-1,2,3,4,5,6,7,8-d8 (20)
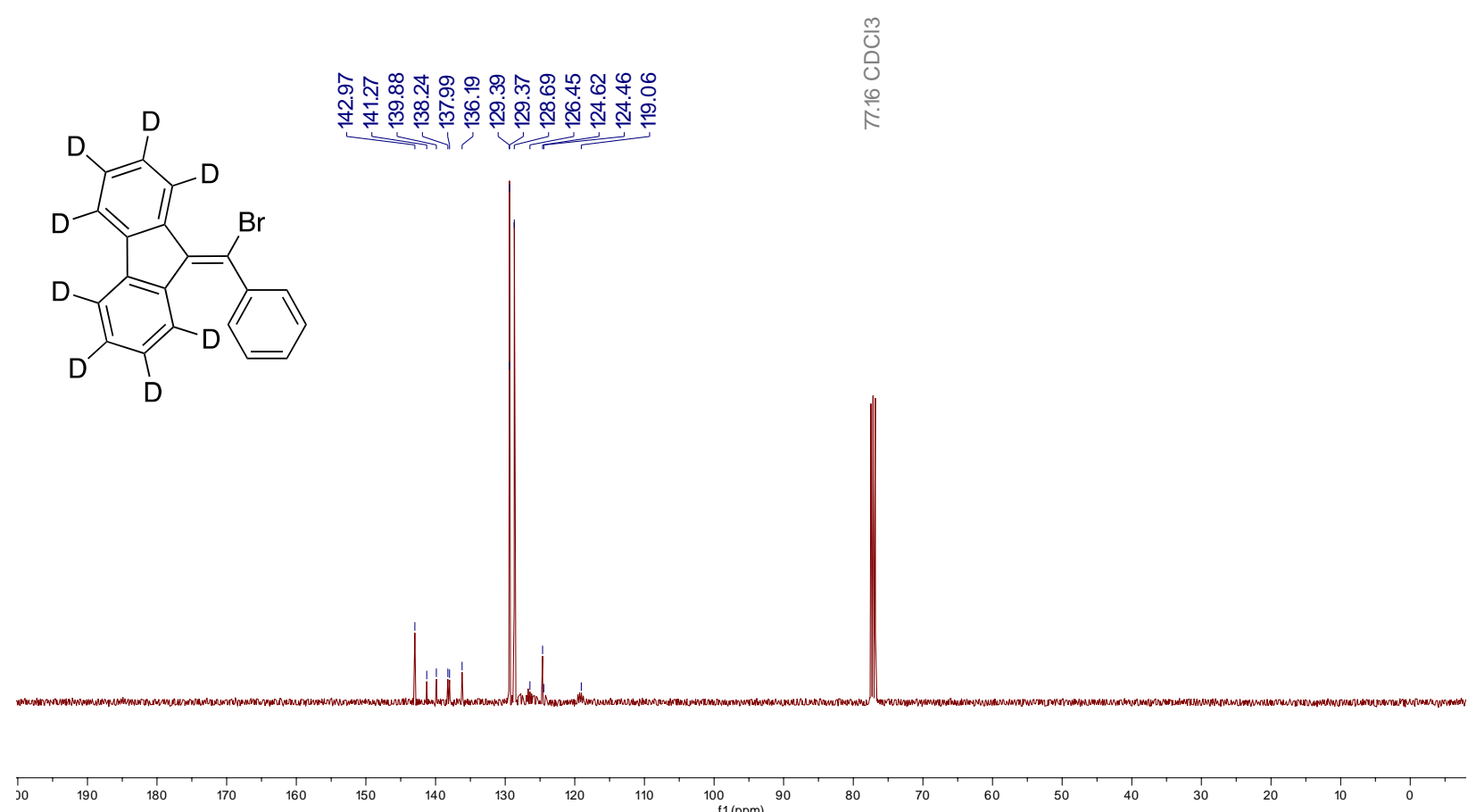

Figure $\mathbf{S 3 1}{ }^{13} \mathrm{C}$ NMR spectrum (151 $\left.\mathrm{MHz}, \mathrm{CDCl}_{3}\right)$ of 9-(bromo(phenyl)methylene)-9Hfluorene-1,2,3,4,5,6,7,8-d8 (20)

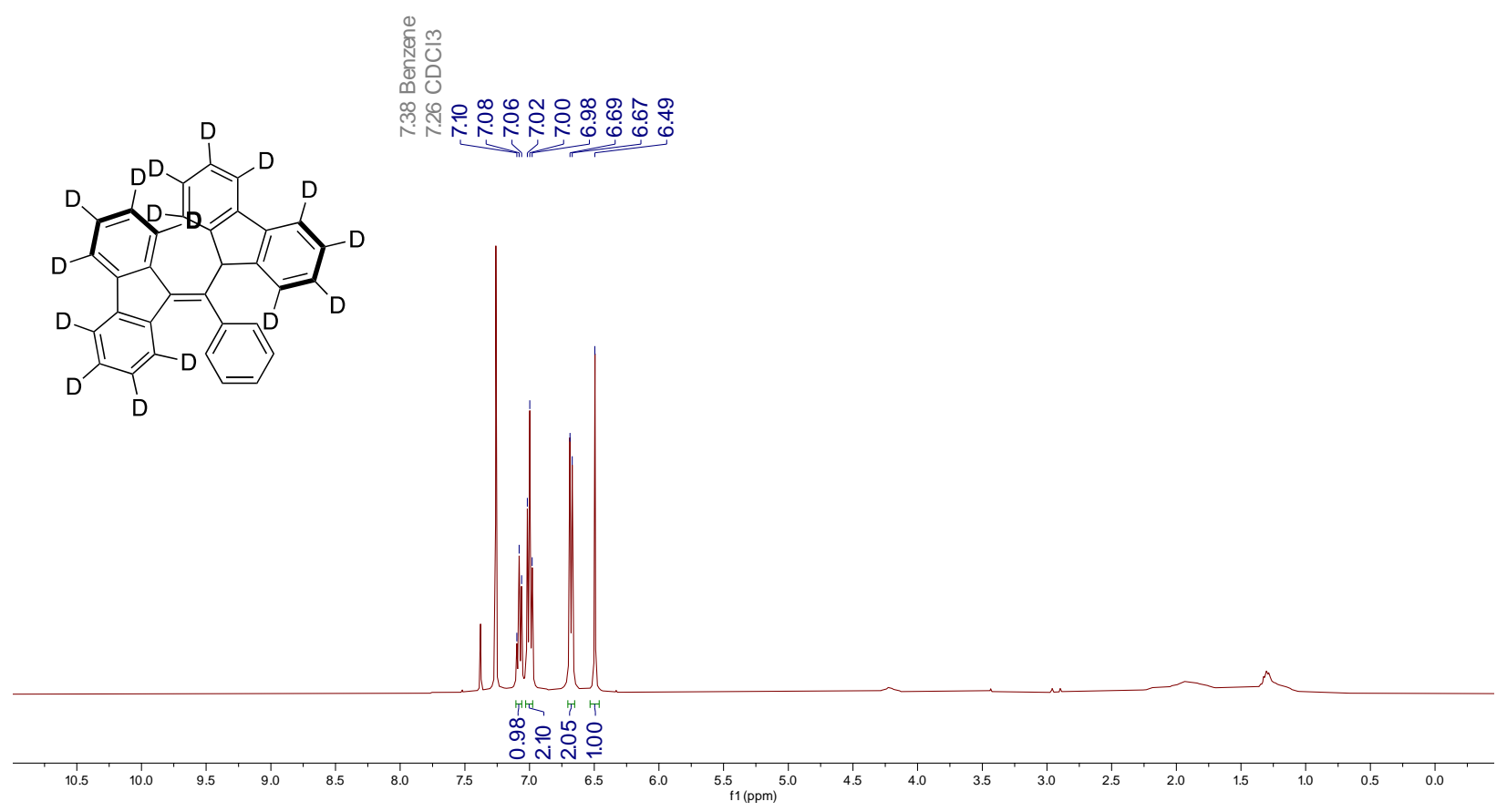


Figure S32 ${ }^{1} \mathrm{H}$ NMR spectrum $\left(600 \mathrm{MHz}, \mathrm{CDCl}_{3}\right)$ of 9-((9H-fluoren-9-yl-1,2,3,4,5,6,7,8d8)(phenyl)methylene)-9H-fluorene-1,2,3,4,5,6,7,8- $d_{8}(21)$
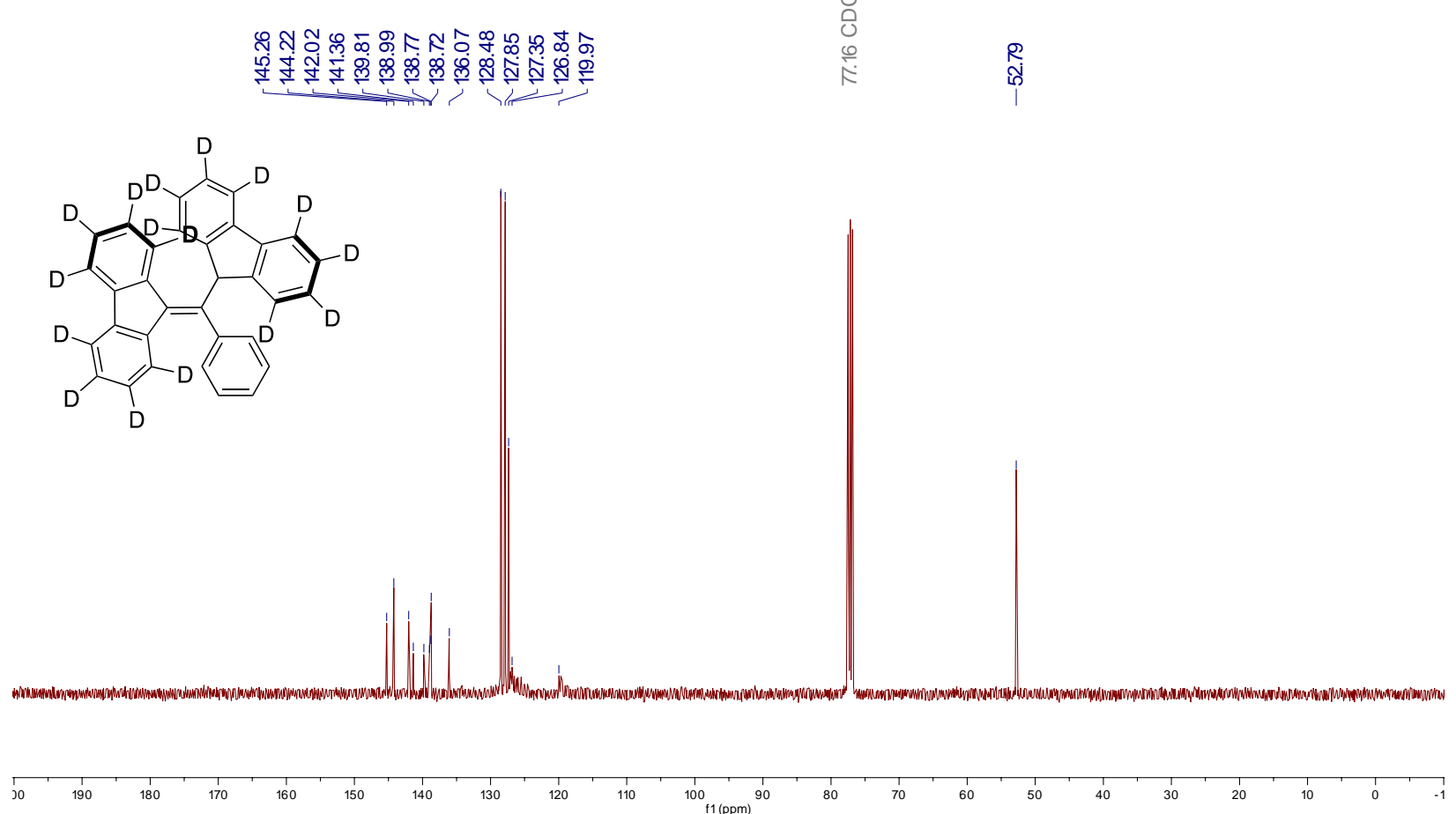

Figure S33 ${ }^{13} \mathrm{C}$ NMR spectrum $\left(151 \mathrm{MHz}, \mathrm{CDCl}_{3}\right)$ of 9-((9H-fluoren-9-yl-1,2,3,4,5,6,7,8d8)(phenyl)methylene)-9H-fluorene-1,2,3,4,5,6,7,8-d8 (21) 


\section{Sample Purity}

(a) stock BDPA from Sigma-Aldrich (b) "purified" BDPA

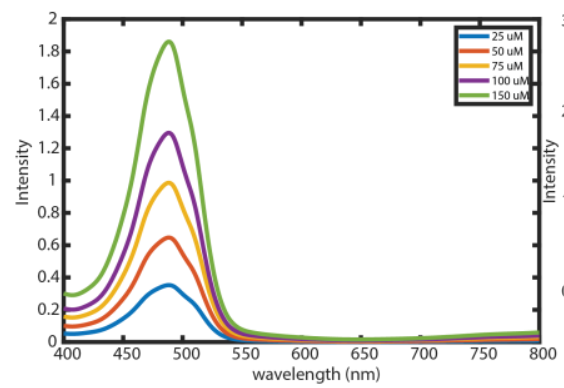

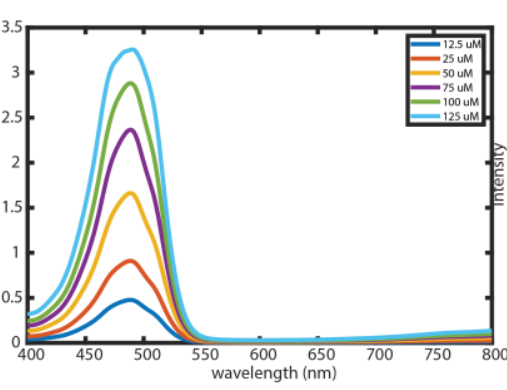

(c) "purified" BDPA +10 days

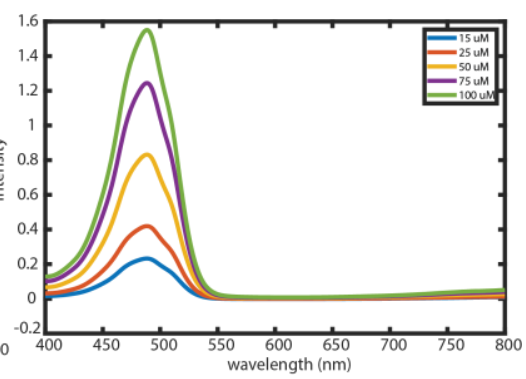

Figure S34. UV-vis measurements at different concentrations for (a) stock $h_{21}$-BDPA (complex with benzene) as purchased from Sigma-Aldrich and used in the presented work, (b) $h_{21}$-BDPA purified via silica gel chromatography, and (c) the same as (b) only measured 10 days later.

\begin{tabular}{|c|c|}
\hline Sample & Extinction coefficient \\
\hline Stock $h_{21}$-BDPA from Sigma-Aldrich & $12930 \pm 650$ \\
\hline "Purified" $h_{21}$-BDPA & $28770 \pm 3300$ \\
\hline "Purified" $h_{21}$-BDPA after 10 days & $16360 \pm 920$ \\
\hline
\end{tabular}

Table S1. Calculated extinction coefficients for $h_{21}$-BDPA based on measurements given in Fig. S34. The stock $h_{21}$-BDPA used in this work is found to possess only about $50 \%$ of active radicals. 


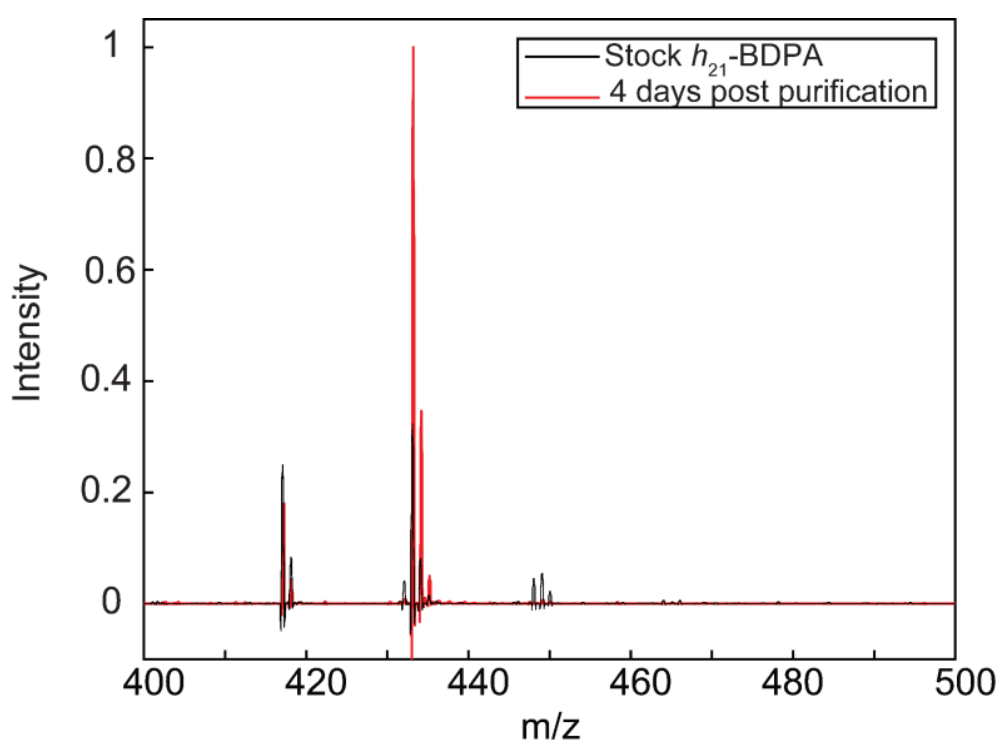

(a)<smiles>c1ccc(C(=C2c3ccccc3-c3ccccc32)C2c3ccccc3-c3ccccc32)cc1</smiles>

(b)

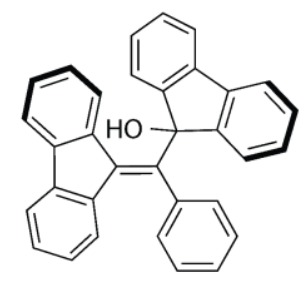

(c)<smiles>O=C(C(=C1c2ccccc2-c2ccccc21)c1ccccc1)C1(O)c2ccccc2-c2ccccc21</smiles>

Molecular Weight: 418.54 Molecular Weight: 434.54 Molecular Weight: 450.54

Figure S35. Matrix assisted laser desorption ionization-time of flight mass spectrometry (MALDI-TOFMS) of Sigma Aldrich of $h_{21}$-BDPA 1:1 in complex with benzene (black) and 4 days after silica gel chromatography purification (red). MALDI-TOF-MS samples were prepared by dissolving $h_{21}$-BDPA into chloroform and spotting onto sample plate. Major species in the MALDI-TOF-MS spectrum are (a) $h_{21^{-}}$ BDPA radical, (b) hydroxylated BDPA, and (c) hydroperoxylated BDPA. Following purification of the sample and removal of benzene, $h_{21}$-BDPA is prone to hydroxylation. 


\section{Radical Concentrations}

EPR signal intensity of DNP samples in a $4 \mathrm{~mm}$ sapphire rotor was determined using a Magnettch ESR5000 spectrometer and double integral (DI) signal intensities were calculated using MATLAB [5]. The quantity of electron spins in $h_{21}$-BDPA, $1,3-\left[\alpha, \beta, \gamma, \delta-d_{16}\right]$-BDPA , $1,3-\left[\alpha, \gamma-d_{8}\right]-$ BDPA, and $1,3-\left[\beta, \delta-d_{8}\right]$-BDPA were determined relative to a standard sample whose concentration and number of electron spins was determined using absolute spin quantification methods on a EMXnano spectrometer. The number of electron spins, $n$, in each DNP sample were determined as $n_{D N P}=\frac{D I_{D N P} \times n_{\text {Standard }}}{D_{\text {Standard }}}$. In Figure $S 36$, the double integrals for each of the four polarizing agents are shown. The concentration was then calculated by measuring the length of sample in the rotor, the uncertainty in length measurement is reflected in the minimum and maximum concentration values in Figure S37.

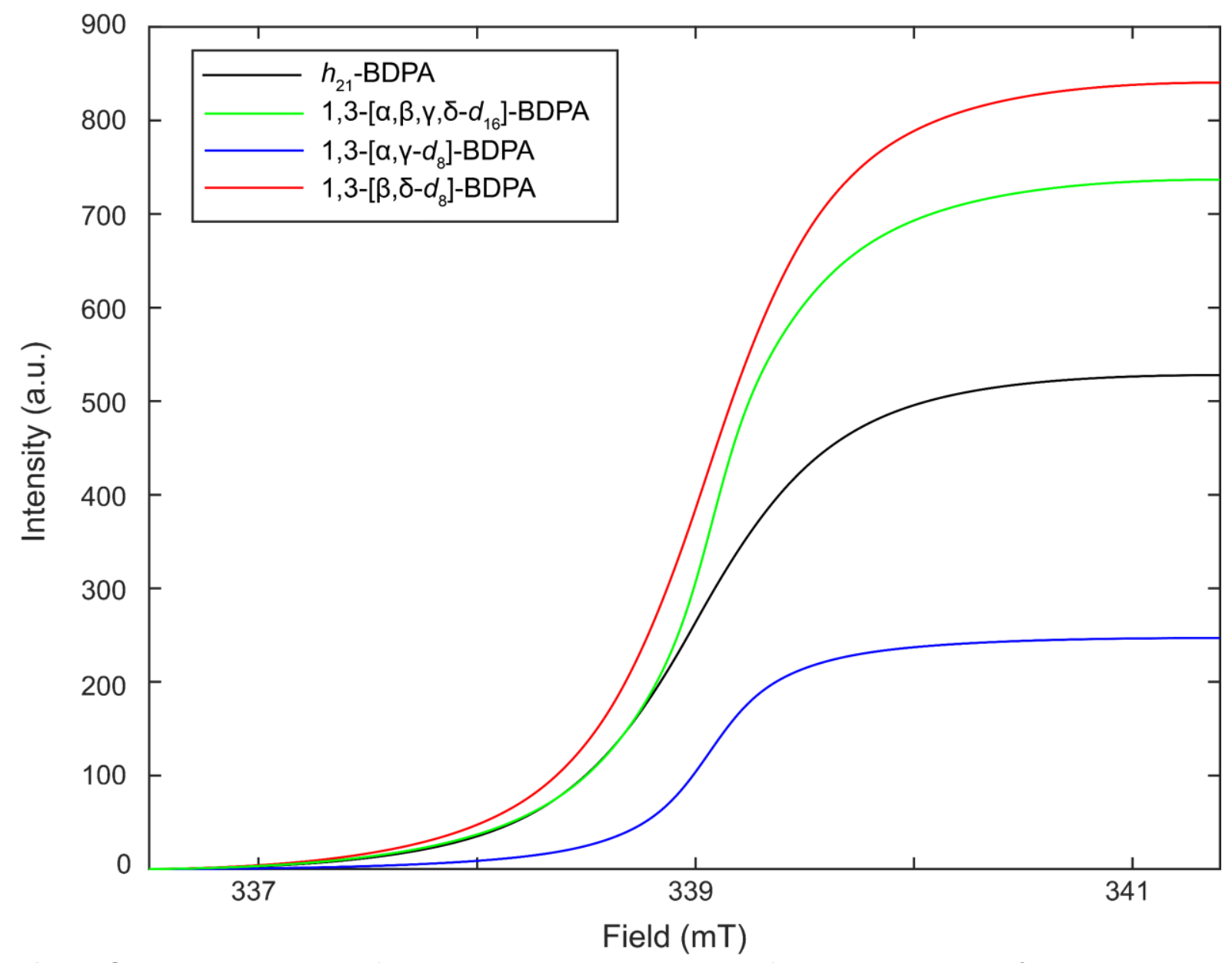

Figure S36. Double Integral of X-Band EPR derivative spectra for $h_{21}-B D P A, 1,3-\left[\alpha, \beta, \gamma, \delta-d_{16}\right]-B D P A$,

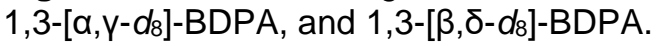




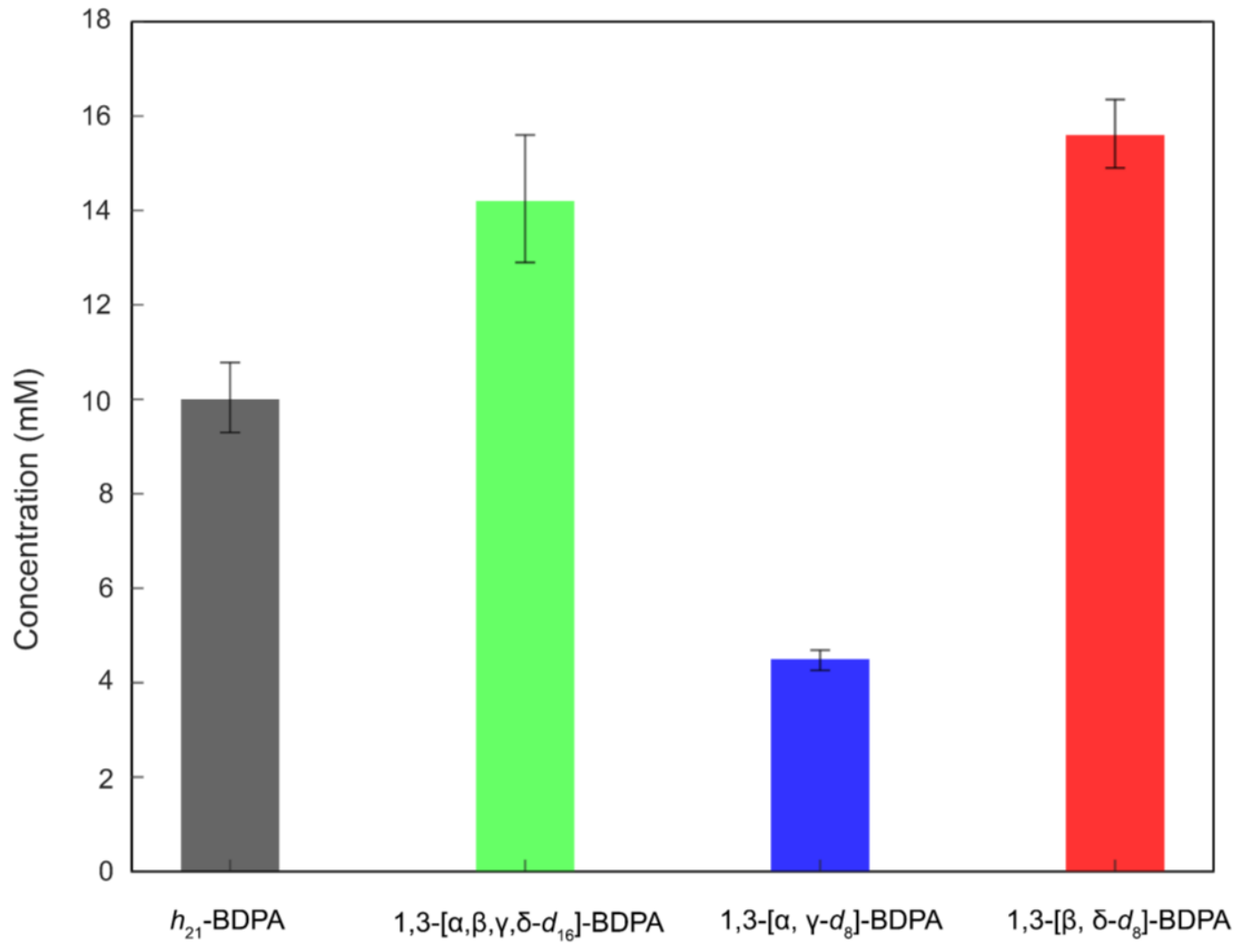

Figure S37. Radical concentrations from X-Band EPR for $h_{21}$-BDPA, 1,3-[ $\left.\alpha, \beta, \gamma, \delta-d_{16}\right]-B D P A, 1,3-\left[\alpha, \gamma^{-}\right.$ $\left.d_{8}\right]$-BDPA, and 1,3-[ $\left[\beta, \delta-d_{8}\right]-B D P A$. Determined concentrations by EPR were $10 \mathrm{mM}, 14.2 \mathrm{mM}, 4.5 \mathrm{mM}$,

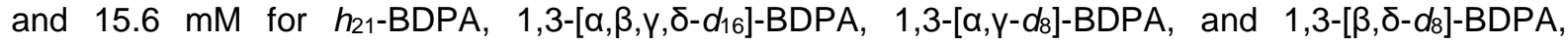
respectively. 


\section{DNP Supporting Information}

All DNP samples were degassed via a freeze-pump-thaw procedure using the homebuilt adapter shown in Figure S38(a). The adapter was 3D printed using a Form3 3D printer using Rigid 4K resin (Formlabs Somerville, MA). The adapter was then epoxied to a 3-way valve, as shown in Figure S38(b), through which vacuum was applied to the rotor directly. The sample was kept immersed in liquid nitrogen while being transferred to a glove bag following 5 freeze-pumpthaw cycles. The rotor was removed from degassing apparatus, the endcap was inserted in a glove bag and the rotor assembly was then immediately inserted into DNP probe precooled to 90 K.

(a)

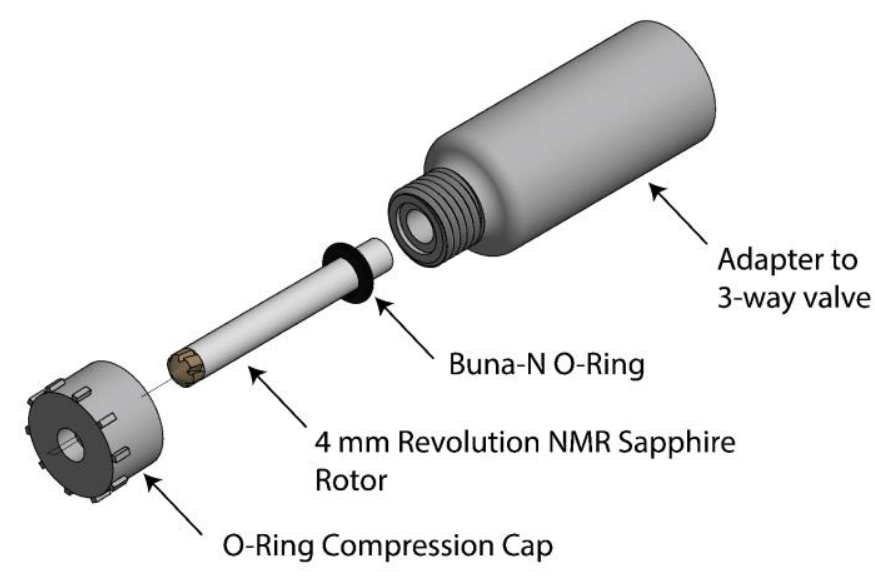

(b)

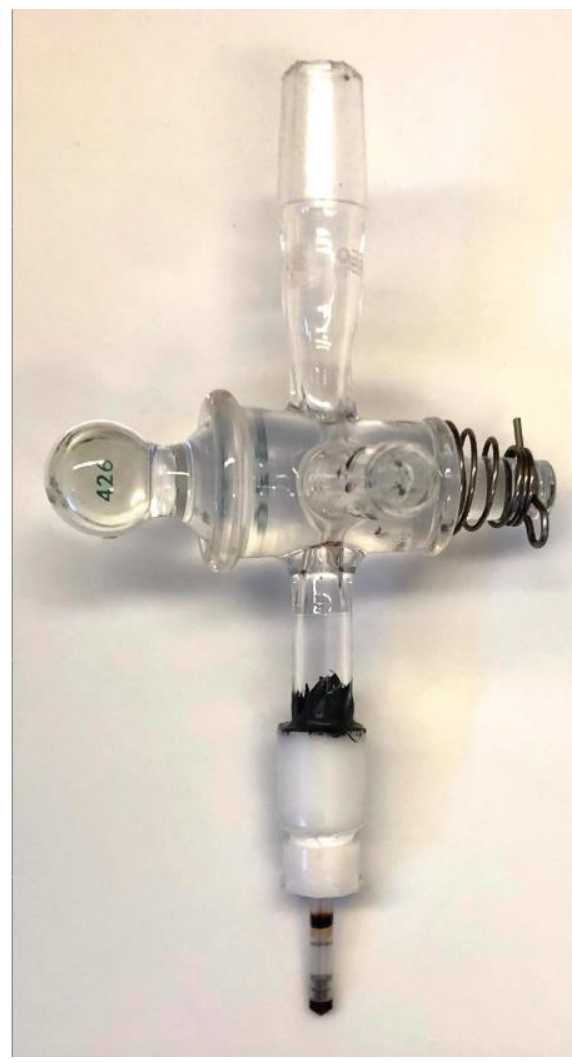

Figure S38. Homebuilt degassing apparatus used for DNP sample preparation. (a) CAD rendering of 3D printed adapter. Sample rotor was sealed against atmosphere using Buna-N O-ring which was observed to maintain sufficient sealing while sample was immersed into liquid nitrogen during freezing and pumping steps. (b) Photograph of assembled adapter epoxied on 3-way valve which enabled connection to vacuum pump in addition to nitrogen gas purge line. 


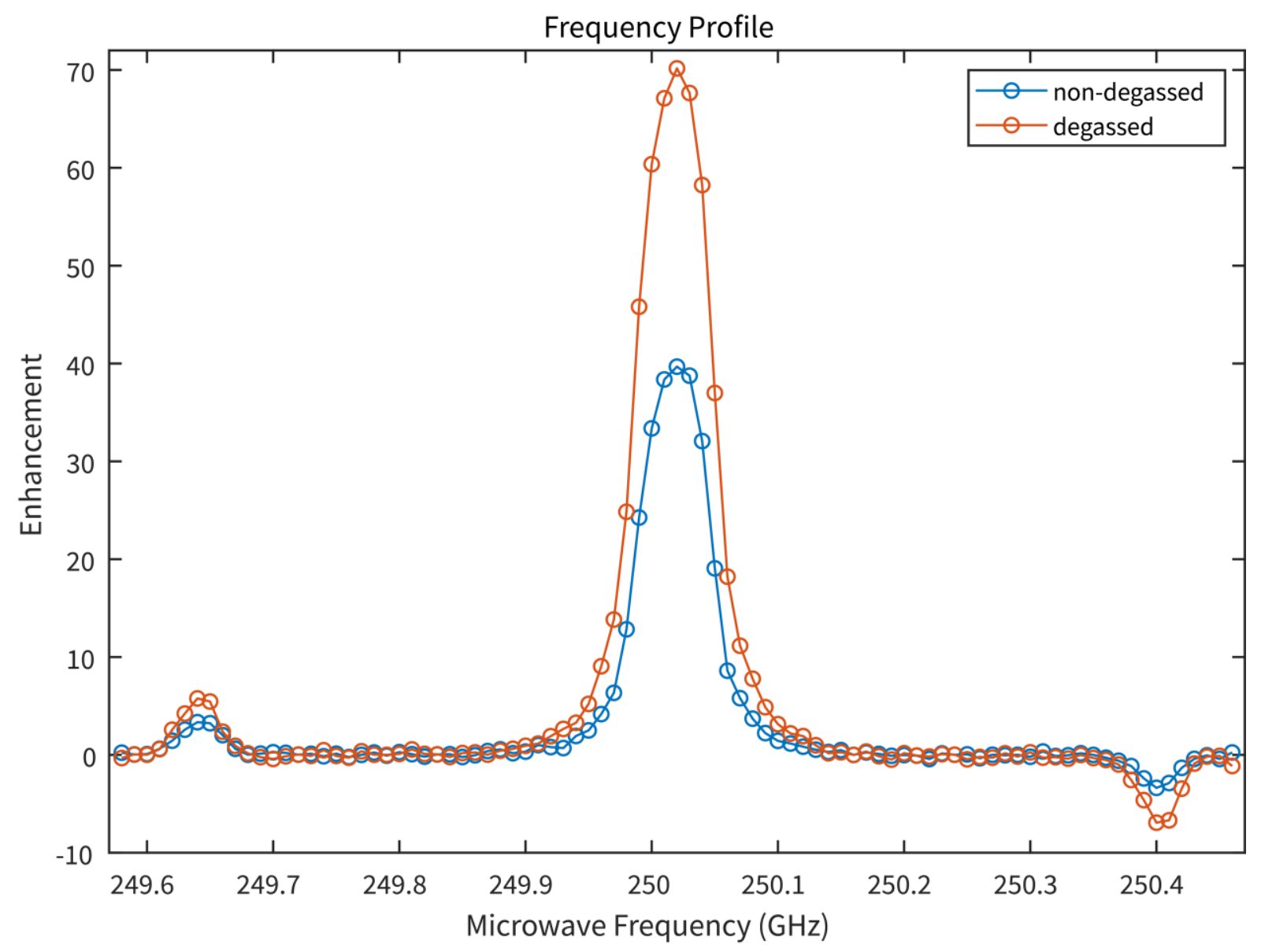

Figure S39. ${ }^{1} \mathrm{H}$ DNP enhancement frequency profiles for $2.5 \mathrm{wt} \% h_{21}$-BDPA in $5 \mathrm{~mol} \% h_{14}$ - and 95 mol\% $d_{14-0 T P}$ matrix with the sample subjected to freeze-pump-thaw degassing (red) or not (blue). The NMR magnetic field strength was $8.92 \mathrm{~T}\left(380 \mathrm{MHz}{ }^{1} \mathrm{H}\right.$ Larmor frequency), the sample temperature was $90 \mathrm{~K}$, and the MAS frequency was $5 \mathrm{kHz}$.

\begin{tabular}{|c|c|c|c|c|c|}
\hline Sample & $\mathbf{T}_{\mathbf{1}} / \mathbf{s}$ & $\mathbf{T}_{\mathbf{B}, \mathbf{O E}} / \mathbf{s}$ & $\mathbf{T}_{\mathbf{B}, \mathbf{S E}} / \mathbf{s}$ & $\mathbf{E n h}$. (OE) & Enh. (SE) \\
\hline Non-degassed & $32.1 \pm 2.2$ & $37.5 \pm 1.5$ & $38.4 \pm 1.9$ & 39.7 & 3.3 \\
\hline Degassed & $42.6 \pm 3.3$ & $43.6 \pm 2.3$ & $42.4 \pm 2.1$ & 70.2 & 5.8 \\
\hline
\end{tabular}

Table S2. Experimentally observed spin-lattice relaxation times $\left(T_{1}\right)$, DNP buildup times with the microwave irradiation at the OE frequency $\left(T_{B, O E}\right)$ or at the positive $S E$ frequency $\left(T_{B, S E}\right)$ are expressed in seconds for $2.5 \mathrm{wt} \% h_{21}$-BDPA in $5 \mathrm{~mol} \% h_{14-}$ and $95 \mathrm{~mol} \% d_{14-0}$ OTP matrix with the sample subjected to freeze-pump-thaw degassing or not. The enhancement values calculated for the OE and the positive SE are also mentioned. The NMR magnetic field strength was $8.92 \mathrm{~T}$ (380 MHz ${ }^{1} \mathrm{H}$ Larmor frequency), the sample temperature was $90 \mathrm{~K}$, and the MAS frequency was $5 \mathrm{kHz}$. 


\section{References}

1. Gandeepan, P., C.-H. Hung, and C.-H. Cheng, Pd-catalyzed double $\mathrm{C}-\mathrm{H}$ bond activation of diaryl ketones for the synthesis of fluorenones. Chemical Communications, 2012. 48(75): p. 9379-9381.

2. Brunetti, F.G., et al., Strain and Hückel Aromaticity: Driving Forces for a Promising New Generation of Electron Acceptors in Organic Electronics. Angewandte Chemie International Edition, 2010. 49(3): p. 532-536.

3. Plater, M.J., S. Kemp, and E. Lattmann, Heterocyclic free radicals. Part 1. 4,5Diazafluorene derivatives of Koelsch's free radical: an EPR and metal-ion complexation study. Journal of the Chemical Society, Perkin Transactions 1, 2000(6): p. 971-979.

4. Haze, O., et al., Water-Soluble Narrow-Line Radicals for Dynamic Nuclear Polarization. Journal of the American Chemical Society, 2012. 134(35): p. 14287-14290.

5. MATLAB R2020a. 2020, The MathWorks, Inc. Natick, Massachusetts. 